Department of Computer Science

\title{
Marginality Approach To Shapley Value In Games With Externalities
}

Oskar Skibski, Tomasz P. Michalak, and Michael Wooldridge

\section{CS-RR-13-08}

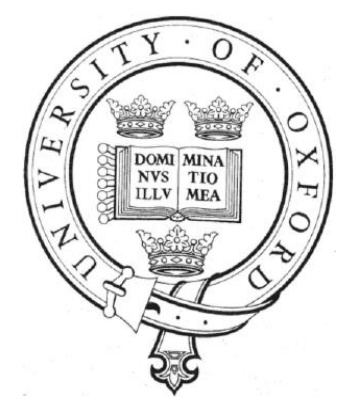

Department of Computer Science, University of Oxford Wolfson Building, Parks Road, Oxford, OX1 3QD 


\title{
MARGINALITY APPROACH TO SHAPLEY VALUE IN GAMES WITH EXTERNALITIES
}

\author{
Oskar Skibski ，Tomasz P. Michalak and Michael Wooldridge ${ }^{1}$
}

One of the long-debated issues in coalitional game theory is how to extend the Shapley value to games with externalities. In particular, when externalities occur, a direct translation of Shapley's axioms does not imply a unique value. In this paper we study the marginality approach to this problem, based on the idea of an $\alpha$ parametrized definition of the marginal contribution, where $\alpha$ is a vector of weights associated with an agent joining/leaving a coalition. We prove that all values that satisfy the direct translation of Shapley's axioms can be obtained using the marginality approach. Moreover, we show that every such value can be uniquely derived using marginality approach by choosing appropriate weights $\alpha$. Next, we analyze how properties of a value translate to the requirements on the definition of the marginal contribution (i.e. weights $\alpha$ ). Building upon this analysis, we show that under certain conditions, two other axiomatizations of the Shapley value (i.e., Young's marginality axiomatization and Myerson's axiomatization based on the concept of balanced contributions), translated to games with externalities using the proper definition of the $\alpha$-parametrized marginal contribution, are equivalent to Shapley's axiomatization.

KEYWORDS: Shapley value, partition function games, axiomatization.

\section{INTRODUCTION}

Fair division of surplus achieved via cooperation is one of the fundamental concerns of coalitional game theory. It is relevant to a wide range of economic and social situations, from sharing the cost of a local wastewater treatment plant, through dividing the annual profit of a joint venture enterprise, to determining power in voting bodies. Assuming that the coalition of all the players (i.e. the grand coalition) forms, Shapley [17] proposed a unique division scheme that meets the following four axioms: Efficiency - all payoff is distributed among the players, Null Player - a player with no influence on payoffs receives nothing, Symmetry - symmetric players obtain the same payoff, and Additivity - i.e. that the division scheme is additive. However, this remarkable result, now called the Shapley value, holds only when one cooperative arrangement does not impose externalities on other arrangements. Such an assumption is clearly untenable in many practical economic situations of interest. For example, on the oligopolistic

\footnotetext{
University of Warsaw

${ }^{1}$ We thank Shmuel Zamir, Sergiu Hart and other participants of the 24th International Conference on Game Theory at Stony Brook University for comments, interesting discussions and encouragement. Tomasz Michalak and Michael Wooldridge were supported by the European Research Council under Advanced Grant 291528 (RACE).
} 
market, joint $\mathrm{R} \& \mathrm{D}$ projects increase the competitive edge of cooperating companies. Similarly, the extent of pollution reduction achieved by an international treaty depends not only on its signatories but also on similar agreements among non-participants. Extending the Shapley value to all such settings has been a subject of ongoing debate in the literature for more than forty years. This is also the focus of our paper.

A natural requirement for a fair division scheme is that it remunerates the players of a coalitional game taking into account their contribution to the surplus generated via cooperation. Indeed, in Shapley's axiomatization, the Null-Player Axiom requires that no share be allocated to players with zero contribution to any possible coalition that could be created in the coalitional game. The key issue, then, is how such contribution should be measured. Although not explicitly, Shapley's Null-Player Axiom relies on the concept of marginal contribution, one of the fundamental notions in economic theory.

In the cooperative-game context, the marginal contribution of a player to a coalition is the difference between the value of this coalition with and without the player. It can be also understood as a loss incurred by the remaining players should the player leave a given coalition. Considering this latter intuition, the Shapley value is defined as the average marginal contribution of a player, taken over all possible ways to dissolve the grand coalition by removing players one after the other in a queue (i.e. permutation) until the empty coalition is obtained. ${ }^{1}$ In any given permutation, the marginal contribution of a particular player is assigned deterministically as it does not play a role in what a player does after leaving a coalition. This is, however, not the case in games with externalities, where the definition of the marginal contribution becomes much more intricate.

When externalities are present, the value of the coalition that a player has left may be influenced by which coalition, if any, this player subsequently joins. In other words, the choice of action after leaving a coalition may result in different values of the player's marginal contribution to it. A way to account for all such values is to assume that a player can choose to join different coalitions with different probabilities - we will denote the set of such probabilities (or weights) by $\alpha$. Then, in games with externalities, the sequential dissolution of the grand coalition according to a given permutation of players can be viewed as a stochastic and not deterministic process. ${ }^{2}$ The marginal contribution of a player is then the difference between the value of the coalition with the player and the expected value of this coalition when the player has left.

In games with externalities, not only the definition of the marginal contribution but also the axiomatization of the value becomes more involved, and it can be easily shown that the standard translation of Shapley's four axioms to games with externalities is not sufficient to obtain a unique value. A number of methods

\footnotetext{
${ }^{1}$ Note that typically the intuition behind the Shapley value is presented as a process of joining coalitions. We adopt a reverse convention as more convenient for games with externalities.

${ }^{2}$ An interesting recent model of coalition formation as a stochastic process, though with no externalities, can be found in [5].
} 
have been developed in the literature to address this issue. Some, such as [16] and [9], managed to obtain uniqueness via modification of some of Shapley's original axioms. Other contributors added new axioms (and sometimes dropped some of the original ones) driving increasingly further away from Shapley's axiomatization. For instance, Grabisch and Funaki used Markovian and Ergodic Axiom and modified Symmetry and the Null-Player Axiom [7]. Yet another method was to build extensions to games with externalities relying on alternative axiomatizations of the original Shapley value, such as Myerson's [13] balanced-contribution axiomatization or Young's [19] monotonicity axiomatization.

In all of the above methods, agents can be remunerated based on their marginal contribution parametrized with $\alpha$. To date, the most general result in this spirit was obtained for the third method, where Fujinaka [6] proved that, for any $\alpha$, Young's monotonicity axiomatization, parametrized with $\alpha$, guarantees a unique value. However, no such study for Shapley's original axiomatization exists in the literature.

Therefore, in this paper, we focus on the first method, that is, we study how Shapley's original axiomatization can be adapted to games with externalities using marginal contribution parametrized with $\alpha$-weigths. We will refer to such an approach as to the "marginality approach".

We begin by proving that, for every value of $\alpha$, Shapley's original axioms of Efficiency, Symmetry, Additivity and the $\alpha$-parametrized Null-Player Axiom yield a unique extension of the Shapley value to games with externalities. We will refer to this value as to $\alpha$-value. The results by [16, 9, 18], focusing on three particular sets of weights $\alpha$, can be considered as special cases of this general theorem. Furthermore, this theorem is a counterpart of Fujinaka's [6] result for Young's axiomatixation. We then extend the analysis of marginality approach as follows.

A fundamental question arising with respect to $\alpha$-value is: which values - either among those already proposed in the literature or any new potential ones - can be defined as an $\alpha$-value? As the key result of our work, we prove the marginality approach encompasses all values that satisfy Shapley's original axiomatization and exactly those.

Next, we analyze how properties of an $\alpha$-value translate into properties of weights $\alpha$. In particular, we focus on the axioms of Weak Monotonicity, Strong Monotonicity, Strong Symmetry, and the Strong Null-Player Axiom.

Weak (Strong) Monotonicity states that if we increase the value of a coalition containing a player, the payoff of this player will not decrease (will increase). We prove that $\alpha$-value satisfies Weak (Strong) Monotonicity if and only if weights $\alpha$ are non-negative (positive).

The next axiom, Strong Symmetry, requires that the value of any coalition has a symmetric influence not only on the payoffs of its members but also on the payoffs of all non-members. We prove that $\alpha$-value satisfies Strong Symmetry if and only if weights $\alpha$ are such that the permutation in which players leave the grand coalition does not affect the probability that a given coalition structure is 
eventually created. We say that weights $\alpha$ satisfying this condition are interlace resistant. As a corollary to this result we have that the average approach of translating the Shapley value to games with externalities, proposed by MachoStadler et al. [10], is a subclass of the marginality approach and is equivalent to the marginality approach used with interlace resistant weights.

The Strong Null-Player Axiom states that a player who does not have an impact on the values of coalitions in the game does not affect the payoff divisionthat is, if we remove a null-player the payoffs from the game will stay the same. We prove that $\alpha$-value satisfies the Strong Null-Player Axiom if and only if weights $\alpha$ are such that the probability of joining a particular coalition depends only on the other coalitions in the coalition structure and not on the coalition that is being left. This condition on weights $\alpha$ we call expansion resistance.

Although interlace and expansion resistance conditions seem arbitrary, they emerge to be key to understanding the relationship between the $\alpha$-parametrized Shapley axiomatization and the Myerson axiomatization based on the concept of balanced contributions extended to games with externalities. In our analysis, we define a game without a player as the difference between the normal game and the game constructed of marginal contributions of this player. Building upon this game, we prove that the $\alpha$-value satisfies Myerson's axioms (Efficiency, $\alpha$ parametrized Balanced Contributions) if and only if $\alpha$ is interlace and expansion resistant.

The remainder of this paper is organized as follows. In next section, we introduce necessary definitions and notations. In Section 3, we formally introduce the marginality approach, prove its generality and show that the $\alpha$-parametrized Shapley axiomatization yields a unique value. In Section 4, we analyze properties of weights $\alpha$. In Section 5, we discuss relationships between different axiomatizations. Related work and conclusions follow.

\section{DEFINITIONS}

Let $N=\{1,2, \ldots, n\}$ be a set of players. A coalition, $S$, is any non-empty subset of $N$. A partition, denoted $P$, is any set of disjoint coalitions whose union is $N$. For technical convenience, we will assume that $\emptyset \in P$ for every partition $P$. A pair $(S, P)$, where $P$ is a partition of $N$ and $S \in P$, is called an embedded coalition. The set of all partitions and the set of all embedded coalitions over $N$ are denoted by $\mathcal{P}(N)$ and $E C(N)$ or, simply, $\mathcal{P}$ and $E C$, when the set of players is clear from the context.

A game $v$ (in partition-function form) is given by a function that associates a real number with every embedded coalition, i.e., $v: E C \rightarrow \mathbb{R}$. A game has externalities if values of (some) coalitions depend on the arrangement of outside agents, that is, for at least one coalition $S$ there exist two partitions $P_{1}, P_{2}$ containing $S$ such that $v\left(S, P_{1}\right) \neq v\left(S, P_{2}\right)$. Otherwise, we say that the game is without externalities. Such games can be represented in characteristic-function form: $\hat{v}: 2^{N} \rightarrow \mathbb{R}$. As is customary in the literature, we assume that the grand 
coalition will form (i.e., the coalition $N$ of all players). Then the outcome of the game (or the value of the game) is a vector that distributes the value of the grand coalition among players.

In various parts of the paper we will use class of simple games $\left\langle e^{(S, P)}\right\rangle_{(S, P) \in E C}$ :

$$
e^{(S, P)}(S, P)=1 \text { and } e^{(S, P)}(\tilde{S}, \tilde{P})=0 \text { otherwise. }
$$

Thus, in a simple game $e^{(S, P)}$ only coalition $S$ in partition $P$ has non-zero payoff.

The set of all permutations of set $S$ will be denoted by $\Omega(S)$. As is common in combinatorics, we identify permutation $\pi \in \Omega(S)$ with a corresponding ordering. Formally, $\pi$ can be understood as a function that assign positions to players, i.e., bijection $\pi:\{1,2, \ldots,|S|\} \rightarrow S$. We will denote the set of agents that appear in permutation $\pi$ after $i$ by $C_{i}^{\pi}$, i.e., $C_{i}^{\pi} \stackrel{\text { def }}{=}\left\{\pi(j) \mid j>\pi^{-1}(i)\right\}$. The concatenation of permutations $\pi_{1}, \pi_{2}$ will be denoted $\pi_{1} \| \pi_{2}$, e.g., $(3,4,1)\|2\| 5=(3,4,1,2,5)$ (for one-element permutation we will omit the brackets).

We use a shorthand notation for set subtraction and set union operations: $N_{-S} \stackrel{\text { def }}{=} N \backslash S$ and $S_{+\{i\}} \stackrel{\text { def }}{=} S \cup\{i\}$. Often, we omit brackets and simply write $S_{+i}$. For partitions, if $P \in \mathcal{P}(N)$, then $P_{-i}$ is a partition of players $N \backslash i$. To model the partition obtained by the transfer of agent $i$ to coalition $T$ in partition $P$, we introduce the following notation:

$$
\tau_{i}^{T}(P) \stackrel{\text { def }}{=} P \backslash\{P(i), T\} \cup\left\{P(i)_{-i}, T_{+i}\right\},
$$

where $P(i)$ denotes $i$ 's coalition in $P$. Finally, the partition obtained by the transfer of group players $S$ to a new coalition will be denoted $P_{[S]}: P_{[S]} \stackrel{\text { def }}{=}$ $\{T \backslash S \mid T \in P\} \cup\{S\}$.

For games without externalities, Shapley [17] famously proved that there exists only one payoff distribution scheme that satisfies four desirable axioms. We state them in the general form, i.e. using the notation for games with externalities, so that their definition will also hold in the remainder of this paper. We will call them a direct translation of Shapley's axioms, or simply, Shapley's axioms:

- Efficiency (the entire available payoff is distributed among agents): $\sum_{i \in N} \varphi_{i}(v)=$ $v(N,\{N, \emptyset\})$ for every game $v$;

- Symmetry (payoffs do not depend on the agents' names): $\varphi(f(v))=f(\varphi)(v)$ for every game $v$ and every bijection $f: N \rightarrow N ;^{3}$

- Additivity (the sum of payoffs in two separate games equals the payoff in a combined game): $\varphi\left(\beta_{1} v_{1}+\beta_{2} v_{2}\right)=\beta_{1} \varphi\left(v_{1}\right)+\beta_{2} \varphi\left(v_{2}\right)$ for all the games

\footnotetext{
${ }^{3}$ Function $f$ is a permutation, but we reserve this word to interpreting a sequence. Formally, $f(S)$ is an image of $S: f(S) \stackrel{\text { def }}{=}\{f(i) \mid i \in S\}$ and a $f(S, P)$ is defined as follows: $f(S, P) \stackrel{\text { def }}{=}$ $(f(S),\{f(T) \mid T \in P\})$. Now, game $v$ and value $\varphi$ are functions, thus $f(v)$ and $f(\varphi)$ are function compositions: $(f(v))(S, P)=v(f(S, P))$ and $f\left(\varphi_{i}\right)=\varphi_{f(i)}$. Intuitively, value of $(S, P)$ in game $f(v)$ equals the value of an embedded coalition obtained by replacing all players $i$ from $(S, P)$ with $f(i)$. For example, if $f$ exchange 1 and 2 , then $f(v)(\{1\},\{\{1\},\{2,3\}\})=$ $v(\{2\},\{\{2\},\{1,3\}\})$.
} 
$v_{1}, v_{2}$ and scalars $\beta_{1}, \beta_{2} \in \mathbb{R},\left(v_{1}+v_{2}\right)(S, P) \stackrel{\text { def }}{=} v_{1}(S, P)+v_{2}(S, P)$ and $(\beta v)(S, P) \stackrel{\text { def }}{=} \beta \cdot v(S, P) ;^{4}$

- Null-Player Axiom (agents that do not have an impact on the value of any coalition should get nothing): $\forall_{(S, P) \in E C, i \in S} v(S, P)-v\left(S_{-i}, \tau_{i}^{T}(P)\right)=0 \Rightarrow$ $\varphi_{i}(v)=0$ for every game $v$ and agent $i \in N$.

The value is defined by the following equation:

$$
S h_{i}(\hat{v})=\frac{1}{|N| !} \sum_{\pi \in \Omega(N)} \hat{v}\left(C_{i}^{\pi} \cup\{i\}\right)-\hat{v}\left(C_{i}^{\pi}\right) .
$$

Shapley presented the following bargaining process. Assume that players enter the grand coalition in a random order. As a player enters, he receives a payoff that equals his marginal contribution to the group of players that he joins (i.e., $v(S \cup\{i\})-v(S)$ when $i$ joins coalition $S)$. Now, the Shapley value is the expected outcome of player's contributions over all orders (permutations).

Whereas in games with no externalities the marginal contribution of a player in a given permutation is deterministic, this is not necessarily so when externalities are present. A general approach to this more complex situation is discussed in the next section.

\section{MARGINALITY APPROACH AND AXIOMS}

In this section we study the marginality approach to extending the notion of the Shapley value to games with externalities. We begin by presenting the origins of this approach, traces of which can be already found in Bolger [2], and which have been then subsequently used by a few authors to develop their particular extensions $[16,9,18]$. Then, in our analysis of the marginality approach, we first generalize these results from the literature and prove that Shapley's original axioms of Efficiency, Symmetry, Additivity and the parametrized Null-Player Axiom always yield a unique extension to games with externalities. Building upon this, we introduce our main theorem, that the marginality approach is universal, i.e. it allows for obtaining all extensions that satisfy Shapley's axiom and exactly those. Finally, we propose a bargaining process which evaluates to such a value.

\footnotetext{
${ }^{4}$ This translation of Additivity is consistent with $[2,10,11,18]$. In the original axiomatization, Shapley used a weaker version: $\varphi\left(v_{1}+v_{2}\right)=\varphi\left(v_{1}\right)+\varphi\left(v_{2}\right)$. In games without externalities, it was enough to imply that the value is linear; thus, the payoff division does not depend on the unit it is calculated with $($ i.e., $\varphi(\beta v)=\beta \varphi(v))$. However, in games with externalities, the weaker version of Additivity combined with Shapley's other three axioms implies that the value can be scaled, but only by rational numbers (see [10] for details). While we are not aware of real-life applications in which irrational values of coalitions occur, for consistency with the literature, we allow irrational numbers in the function domain; thus, we strengthen Additivity by the linearity condition. However, we retain the name Additivity, as this is a natural translation of this axiom to games with externalities.
} 
Let us start by considering the following example of a 3-player game with externalities, where it is easily visible that Shapley's original axiomatization does not imply a unique value.

Example 1 Let $N=\{1,2,3\}$ and let the partition function $v$ be defined as follows: $v(\{1,2\},\{\{1,2\},\{3\}, \emptyset\})=a, v(\{2\},\{\{1\},\{2\},\{3\}, \emptyset\})=b, v(\{2\},\{\{1,3\}$, $\{2\}, \emptyset\})=c$, and $v(S, P)=0$ for all the remaining embedded coalitions. Now, let us consider a payoff of player 1 in this game that we will denote $\varphi_{1}(v)$. This payoff, according to the extensions of the Shapley value to games with externalities by Pham do and Norde [16], McQuillin [11], Bolger [2], Macho-Stadler et al. [10], and Hu and Yang [9], respectively, is as follows:

$$
\varphi_{1}(v)= \begin{cases}\frac{1}{6}(a-b) & \text { in the case of Pham do and Norde } \\ \frac{1}{6}(a-c) & \text { in the case of McQuillin } \\ \frac{1}{6}\left(a-\frac{b+c}{2}\right) & \text { in the case of Bolger and Macho-Stadler et al. } \\ \frac{1}{6}\left(a-\frac{3 b+2 c}{5}\right) & \text { in the case of Hu and Yang }\end{cases}
$$

As can be seen, although each of these extensions satisfies the direct translation of all four original axioms to games with externalities, ${ }^{5}$ they yield very different payoffs.

The main challenge when constructing a value for games with externalities comes from the fact that it is not straightforward to evaluate the role played by particular players in a setting where evaluating coalitions can be ambiguous, i.e., where embedded coalitions may have different values depending on the partition they are embedded within. All extensions in the literature, including the ones in Example 1, are, in fact, methods to address this problem. For instance, MachoStadler et al. proposed to assume that the value of coalition $S$ is its weighted averaged value over all possible partitions $(S, P)$. The marginality approach is a more generic method to tackle this problem. As a matter of fact, we prove that the average approach by Macho-Stadler et al. is the spacial-case of the marginality approach.

The marginality approach aims at extending the notion of the Shapley value with an axiomatization which is as close to the original one as possible. Whereas, as we have seen in Section 2, the translation of Efficiency, Symmetry and Additivity to games with externalities is straightforward, this is not entirely so with the Null-Player Axiom. In the direct (or strict) translation of this axiom, a player is called a null-player if he never has an impact on the value of any coalition. It means that all his transfers outside a given coalition should not change the value of this coalition.

Example 2 In Example 1, player 1 is a null-player in this sense if $a=b=c$. This is because, under this assumption, we have that the marginal contribution of 1 to $\{1,2\}$ in $\{\{1,2\},\{3\}, \emptyset\}$ considering the transfer to coalition $\{3\}$ is 0 as $v(\{1,2\},\{\{1,2\},\{3\}, \emptyset\})-v(\{1\},\{\{1\},\{2,3\}, \emptyset\})=a-c$. Analogously, creating

\footnotetext{
${ }^{5}$ See below for discussion on the Null-Player Axiom.
} 
a new coalition (i.e., transferring to an empty one) also yields no contribution. Furthermore, it is not difficult to observe that this holds for any other marginal contribution associated with a transfer of player 1 within any partition as payoffs before and after such a transfer equal zero.

We will call marginal contributions associated with a given transfer within a partition (such as those considered in Example 2) elementary marginal contributions.

Now, the marginality approach is based on the observation that, given a partition, although particular transfers may change the value of the embedded coalition, the overall marginal contribution in this partition may still equal zero. For instance, let us set $b=a+r$ and $c=a-r$ in Example 1. Despite the fact that elementary marginal contributions are non-zero, the overall marginal contribution is zero, if we assume that both transfers are evaluated equally. It is, then, a less strict translation of the Null-Player Axiom in which we require that the overall marginal contribution is zero but not necessarily elementary marginal contributions.

It immediately follows from the above analysis that to specify the marginal contribution in the marginality approach, one needs to indicate which transfers are considered and in which proportion. Although, in theory, any function of elementary marginal contributions is admissible, affine ${ }^{6}$ combinations of elementary marginal contributions are enough to obtain every value that meets Shapley's axioms (see Theorem 2 for details):

$$
\left[m c_{i}^{\alpha}(v)\right](S, P) \stackrel{\text { def }}{=} \sum_{T \in P_{-S}} \alpha_{i}\left(S_{-i}, \tau_{i}^{T}(P)\right)\left[v(S, P)-v\left(S_{-i}, \tau_{i}^{T}(P)\right)\right],
$$

where $\alpha_{i}:\{(S, P) \in E C \mid i \notin S\} \rightarrow \mathbb{R}$ denotes weights of a given transfer under the assumptions that:

(a) $\alpha_{i}(S, P)=\alpha_{f(i)}(f(S), f(P))$ for every bijection $f: N \rightarrow N$ and $(S, P) \in$ $E C$ such that $i \notin S$ (to satisfy Symmetry); and

(b) $\sum_{T \in P_{-S}} \alpha_{i}\left(S_{-i}, \tau_{i}^{T}(P)\right)=1$ for every $(S, P) \in E C$ such that $i \in S$ (for normalization).

Note that $\alpha_{i}(S, P)$ is the weight associated with $i$ 's transfer from coalition $S_{+i}$ that results in partition $P$. For example, the weight of 1 's transfer from $(\{1,2\},\{\{1,2\},\{3\}, \emptyset\})$ to $\{3\}$ is denoted by $\alpha_{1}(\{2\},\{\{2\},\{1,3\}, \emptyset\})$. The definition of marginal contribution based on the weights $\alpha$ will be called $\alpha$-marginality. ${ }^{7}$

Now, we define the $\alpha$ version of the Null-Player Axiom (parametrized by weighting $\alpha$ ) as follows:

\footnotetext{
${ }^{6}$ Linear combination $\sum \alpha_{i} x_{i}$ is called affine when weights sum up to one: $\sum \alpha_{i}=1$.

${ }^{7}$ For the generality of the approach, we allow negative weights. However, we show in Section 4.2 that negative weights produce non-monotonic values; thus, we believe that they should not be used. Non-negative weights have a natural interpretation as the probability of transfer and we will refer to this intuition freely.
} 
- $\alpha$-Null-Player Axiom (agents that do not contribute to the value of any coalition should get nothing): $\forall_{(S, P) \in E C, i \in S}\left[m c_{i}^{\alpha}(v)\right](S, P)=0 \Rightarrow \varphi_{i}(v)=$ 0 for every game $v$ and agent $i \in N$.

The theorem below states that Shapley's $\alpha$-parametrized axiomatization, that is, Efficiency, Symmetry, Additivity, and $\alpha$-Null-Player Axiom, is enough to obtain uniqueness for every $\alpha$ and provides a formula for the value. Defining an extension of the Shapley value to games with externalities with such strengthening constitutes the marginality approach.

Before we proceed, let us introduce an important notion of weights composition. Let $\operatorname{pr}_{\pi}^{\alpha}(S, P) \stackrel{\text { def }}{=} \prod_{i \in N_{-S}} \alpha_{i}\left(S \cup C_{i}^{\pi}, P_{\left[S \cup C_{i}^{\pi}\right]}\right)$ for permutations $\pi \in$ $\Omega(N \backslash S)$. Thus, if players $N \backslash S$ leave the grand coalition in order $\pi$ and form partition $P \backslash S$, then $\operatorname{pr}_{\pi}^{\alpha}(S, P)$ is the product of weights associated with these transfers. For example, for permutation $\pi=(1,2,3)$, we have:

$$
\begin{aligned}
\operatorname{pr}_{\pi}^{\alpha}(\{4\},\{\{4\},\{1,2\},\{3\}, \emptyset\})= \\
=\alpha_{1}(\{2,3,4\},\{\{2,3,4\},\{1\}, \emptyset\}) \cdot \alpha_{2}(\{3,4\},\{\{3,4\},\{1,2\}\}) \\
\cdot \alpha_{3}(\{4\},\{\{4\},\{1,2\},\{3\}, \emptyset\})
\end{aligned}
$$

Now, we will prove the following theorem:

Theorem 1 There exists a unique value that satisfies Efficiency, Symmetry, Additivity and $\alpha$-Null-Player Axiom for every $\alpha{ }^{8}$ Moreover, it satisfies the following formula:

$$
\varphi_{i}^{\alpha}(v) \stackrel{\text { def }}{=} \frac{1}{|N| !} \sum_{\pi \in \Omega(N)} \sum_{P \in \mathcal{P}} \operatorname{pr}_{\pi}^{\alpha}(\emptyset, P) \cdot\left[v\left(C_{i}^{\pi} \cup\{i\}, P_{\left[C_{i}^{\pi} \cup\{i\}\right]}\right)-v\left(C_{i}^{\pi}, P_{\left[C_{i}^{\pi}\right]}\right)\right] .
$$

Proof. First we prove that $\varphi^{\alpha}$ satisfies all four axioms. Then, we show that this is the only such value.

Part 1: We examine the axioms one by one. First, let us consider Efficiency. For any permutation $\pi$ and partition $P$, the elementary marginal contributions add up to $v(N,\{N, \emptyset\})$; thus:

$$
\begin{aligned}
\sum_{i \in N} \varphi_{i}^{\alpha}(v) & =\frac{1}{|N| !} \sum_{\pi \in \Omega(N)} \sum_{P \in \mathcal{P}} p r_{\pi}^{\alpha}(\emptyset, P) \sum_{i \in N}\left[v\left(C_{i}^{\pi} \cup\{i\}, P_{\left[C_{i}^{\pi} \cup\{i\}\right]}\right)-v\left(C_{i}^{\pi}, P_{\left[C_{i}^{\pi}\right]}\right)\right] \\
& =\frac{1}{|N| !} \sum_{\pi \in \Omega(N)} \sum_{P \in \mathcal{P}} p r_{\pi}^{\alpha}(\emptyset, P) \cdot v(N,\{N, \emptyset\})=v(N,\{N, \emptyset\}),
\end{aligned}
$$

where the last transformation comes from the fact that weights of all partitions sum up to one for every permutation: $\sum_{P \in \mathcal{P}} \operatorname{pr}_{\pi}^{\alpha}(\emptyset, P)=1$.

Formula (1) clearly shows that the value satisfies Symmetry and Additivity. Regarding Symmetry, it does not favor any player, hence permutation of coalitions' values will permute payoffs accordingly. The value is additive as $\varphi_{i}^{\alpha}\left(v_{1}+v_{2}\right)$ can be split into two expressions representing $\varphi_{i}^{\alpha}\left(v_{1}\right)$ and $\varphi_{i}^{\alpha}\left(v_{2}\right)$.

\footnotetext{
${ }^{8}$ Although we assumed a stronger definition of Additivity, our proof is based only on the weaker version that does not force linearity: $\varphi\left(v_{1}+v_{2}\right)=\varphi\left(v_{1}\right)+\varphi\left(v_{2}\right)$. Consequently, the theorem also holds for the weaker version.
} 
To see that $\varphi^{\alpha}$ satisfies the $\alpha$-Null-Player Axiom let us calculate the weight of a given elementary marginal contribution $v(S, P)-v\left(S_{-i}, \tau_{i}^{T}(P)\right)$ using formula (1). A transfer from $(S, P)$ occurs only in permutations where players from $N \backslash S$ (and only them) appear before player $i$. Let us assume that they appear in order $\pi \in \Omega(N \backslash S)$. Then, regardless of the rest of the permutation, the elementary marginal contribution under consideration is multiplied by product of weights $\operatorname{pr}_{\pi}^{\alpha}(S, P)$. The transfer to coalition $T$ causes this product to be multiplied by $\alpha_{i}\left(S_{-i}, \tau_{i}^{T}(P)\right)$. Finally, we observe that the permutation and the arrangement of the remaining players does not have an impact on the value (there are $(|S|-1)$ ! such permutations). This is because, for a given permutation, the sum of products of remaining transfers over all possible partitions sum up to one. Now, if we collect all transfers from a given embedded coalition $(S, P)$ we get the following formula:

$$
\varphi_{i}^{\alpha}(v)=\sum_{(S, P) \in E C, i \in S} \frac{(|S|-1) !}{|N| !} \sum_{\pi \in \Omega\left(N_{-S}\right)} p r_{\pi}^{\alpha}(S, P) \cdot\left[m c_{i}^{\alpha}(v)\right](S, P) .
$$

Part 2: Next, we will show that $\varphi^{\alpha}$ is the only value which satisfies all four of Shapley's original axioms. To this end, let us recall the class of simple games $e^{(S, P)}$. This class forms the basis of the game space, i.e., every game can be defined as a linear combination of games $e^{(S, P)}: v=\sum_{(S, P) \in E C} v(S, P) \cdot e^{(S, P)}$. Based on Additivity, we have $\varphi(v)=\sum_{(S, P) \in E C} \varphi\left(v(S, P) \cdot e^{(S, P)}\right)$; thus, it is enough to prove that the axioms imply a unique value in simple game $e^{(S, P)}$ (multiplied by a scalar). For this purpose, we will use the reverse induction on the size of $S$ : we will show that the value of game $e^{(S, P)}$ can be calculated from the values of simple games for bigger coalitions: $e^{(\tilde{S}, \tilde{P})}$ where $|\tilde{S}|>|S|$. Our base case when $|S|=|N|$ comes from the Efficiency and Symmetry: $\varphi_{i}\left(c \cdot e^{(N,\{N, \emptyset\})}\right)=\frac{c}{|N|}$ for every $i$.

First, let $(S, P)$ be any embedded coalition and assume that $i \notin S$. Let us consider game $\tilde{v}$ combined from two simple games:

$$
\tilde{v}=c \cdot\left[\alpha_{i}(S, P) \cdot e^{\left(S_{+i}, \tau_{i}^{S}(P)\right)}+e^{(S, P)}\right]
$$

It is easy to observe that agent $i$ 's marginal contribution to $\left(S_{+i}, \tau_{i}^{S}(P)\right)$ equals zero, as with all other marginal contributions. Thus, from Null-Player Axiom $\varphi_{i}(\tilde{v})=0$ and from Additivity:

$$
\varphi_{i}\left(c \cdot e^{(S, P)}\right)=-\varphi_{i}\left(c \cdot \alpha_{i}(S, P) \cdot e^{\left(S_{+i}, \tau_{i}^{S}(P)\right)}\right),
$$

if $i \notin S$.

Now, let us assume otherwise, i.e., that $i \in S$ and $|S|<|N|$ (we already considered simple game $e^{(N,\{N, \emptyset\})}$. We have that $v(N,\{N, \emptyset\})=0$. From Efficiency, we can evaluate the sum of payoffs of agents from $S$ as the opposite number to the sum of payoffs of outside agents $\left(-\sum_{j \notin S} \varphi_{j}\left(c \cdot e^{(S, P)}\right)\right)$. This sum, in turn, 
can be calculated with formula (3). Now, based on Symmetry, all agents from $S$ divide their joint payoff equally:

if $i \in S$.

$$
\begin{aligned}
\varphi_{i}\left(c \cdot e^{(S, P)}\right) & =\frac{1}{|S|} \sum_{k \in S} \varphi_{k}\left(c \cdot e^{(S, P)}\right)=-\frac{1}{|S|} \sum_{j \notin S} \varphi_{j}\left(c \cdot e^{(S, P)}\right) \\
& =\frac{1}{|S|} \sum_{j \notin S} \varphi_{j}\left(c \cdot \alpha_{j}(S, P) \cdot e^{\left(S_{+j}, \tau_{j}^{S}(P)\right.}\right),
\end{aligned}
$$

Thus, we provided two recursive equations for $\varphi_{i}\left(c \cdot e^{(S, P)}\right)$ for both cases: $i \in S$ and $i \notin S$. This concludes our proof.

The marginality approach may seem arbitrary, that is, there may exist other values that satisfy Shapley's axiomatization but that cannot be uniquely derived from $\alpha$-parametrized Shapley's axiomatization. This is, however, not the case. The next theorem states that the marginality approach encompasses all values that satisfy Shapley's axiomatization and exactly those.

Theorem 2 Value $\varphi$ can be obtained using marginality approach if and only if it satisfies Efficiency, Symmetry, Additivity and the Null-Player Axiom.

Proof. In the proof of Theorem 1 we showed that every value obtained using marginality approach satisfies all four axioms. Thus, values that do not satisfy these axioms cannot be obtained using marginality approach.

Assume that $\varphi$ satisfies all four axioms. We will prove that there exists weighting $\alpha$ such that $\varphi$ satisfies also $\alpha$-Null-Player Axiom. Based on Theorem 2 this will conclude the proof, as there exists only one value which satisfies Efficiency, Symmetry, Additivity and $\alpha$-Null-Player Axiom. First, let us decompose game $v$ into linear combination of simple games: $v=\sum_{(S, P) \in E C} v(S, P) \cdot e^{(S, P)}$. Based on Additivity, we have that:

$$
\varphi_{i}(v)=\sum_{(S, P) \in E C} v(S, P) \cdot \varphi_{i}\left(e^{(S, P)}\right) .
$$

Now, let $(\tilde{S}, \tilde{P}) \in E C$ be any embedded coalition such that $i \in \tilde{S}$. Consider game $\tilde{v}=e^{(\tilde{S}, \tilde{P})}+\sum_{T \in \tilde{P}_{-\tilde{S}}} e^{\left(\tilde{S}_{-i}, \tau_{i}^{T}(\tilde{P})\right)}$ (i.e., only $(\tilde{S}, \tilde{P})$ and embedded coalitions obtained by transfer of $i$ outside $\tilde{S}$ have non-zero values). Player $i$ is a nullplayer in $\tilde{v}$; thus, from the Null-player Axiom we have that $\varphi_{i}(\tilde{v})=0$ and from Additivity that $\varphi_{i}\left(e^{(\tilde{S}, \tilde{P})}\right)=-\sum_{T \in \tilde{P}_{-\tilde{S}}} \varphi_{i}\left(e^{\left(\tilde{S}_{-i}, \tau_{i}^{T}(\tilde{P})\right)}\right)$. As $(\tilde{S}, \tilde{P}) \in E C$ has been chosen arbitrarily, it holds for every $(S, P)$ such that $i \in S$; thus, we can transform equation (4) as follows:

$$
\begin{aligned}
\varphi_{i}(v) & =\sum_{(S, P) \in E C, i \in S}\left[v(S, P) \cdot \varphi_{i}\left(e^{(S, P)}\right)+\sum_{T \in P_{-S}} v\left(S_{-i}, \tau_{i}^{T}(P)\right) \varphi_{i}\left(e^{\left(S_{-i}, \tau_{i}^{T}(P)\right)}\right)\right] \\
& =\sum_{(S, P) \in E C, i \in S} \sum_{T \in P_{-S}}-\varphi_{i}\left(e^{\left(S_{-i}, \tau_{i}^{T}(P)\right)}\right)\left[v(S, P)-v\left(S_{-i}, \tau_{i}^{T}(P)\right)\right] .
\end{aligned}
$$


Now, we can define $\alpha_{i}(S, P)=-\frac{\varphi_{i}\left(e^{\left(S_{-i}, \tau_{i}^{T}(P)\right)}\right)}{\varphi_{i}\left(e^{(S, P)}\right)}$ : these are proper weights, as they sum up to one for every $(S, P)$ and-based on Symmetry-are symmetrical $\left(\varphi_{i}\left(e^{(S, P)}\right)=\varphi_{\pi(i)}\left(e^{(\pi(S), \pi(P))}\right)\right.$ for every $\left.(S, P) \in E C\right)$. The last transformation shows that $\varphi$ satisfies the $\alpha$-Null-Player Axiom:

$$
\begin{aligned}
\varphi_{i}(v) & =\sum_{(S, P) \in E C, i \in S} \varphi_{i}\left(e^{(S, P)}\right) \sum_{T \in P_{-S}} \alpha_{i}(S, P)\left[v(S, P)-v\left(S_{-i}, \tau_{i}^{T}(P)\right)\right] \\
& =\sum_{(S, P) \in E C, i \in S} \varphi_{i}\left(e^{(S, P)}\right)\left[m c_{i}^{\alpha}(v)\right](S, P)
\end{aligned}
$$

To gain the intuition how weights $\alpha$ impact the value assigned to a player, we present the bargaining process that would produce the value as its expected outcome. To this end, we reverse the process presented by Shapley for the value for games with no externalities and, additionally, investigate partitions of players outside the grand coalition. For clarity of presentation, we limit ourselves to positive weights which can be interpreted as the probability of a transfer to occur.

Assume that players leave a grand coalition in a random order and divide themselves into groups outside. In each step, one player departs and, with the probability given by $\alpha$, enters an existing group outside, or forms a new group. As the result of the leave, the player is granted with his elementary marginal contribution, i.e., with the loss of a coalition he left. Now, the value obtained using marginality approach with weights $\alpha$ is the expected outcome of the player's contribution. $^{9}$

To date in the literature, there have been proposed five particular weights $\alpha$, that is, definitions of marginal contributions $([2,16,10,9,18]$. We will discuss all of them in Section 6, but to illustrate marginality approach we will now present weights considered by Macho-Stadler et al.:

$$
\alpha_{i}^{M S t}(S, P)=\frac{\left|P(i)_{-i}\right|}{|N|-|S|+1}
$$

under the convention that $|\emptyset|=1$. According to this definition, the effects of transfers to bigger coalitions are taken with higher weights. Thus, if we look at the weights $\alpha$ as probabilities, this definition states that a player is more likely to transfer to a bigger coalition than to a smaller one. Interestingly, this means that the formation of a given partition in the bargaining process corresponds to the Chinese restaurant process, known in the field of probability theory.

We have just shown that the marginality approach restricts all payoff division schemes to those that satisfy Shapley's axioms, i.e., $\alpha$-values. In the next sec-

\footnotetext{
${ }^{9}$ To include negative weights in this process, one has to assign to players' transfers weights instead of probabilities. A composition of such weights for a given permutation will constitute a weight of the resulting partition. A marginal contribution in a given permutation should be calculated as a sum of all possible elementary marginal contributions multiplied by corresponding weights.
} 
tion, we will analyze how some desirable properties of an $\alpha$-value translate into properties of weights $\alpha$.

\section{PROPERTIES OF WEIGHTS}

In the marginality approach, we concentrate on the weights associated with transfers. It leads to the natural question of how weights will affect the properties of the resulting value, and what condition have to be met by the weights to obtain a value with the given properties.

In this section, we provide certain links between properties of an $\alpha$-value and the properties of $\alpha$-weights. We begin by identifying insignificant weights, that is, weights which do not have an impact on a value. Then, we show how the axioms of Weak Monotonicity, Strong Monotonicity, Strong Symmetry, and the Strong Null-Player Axiom translate into properties of significant weights $\alpha$.

\subsection{Significant and Insignificant Weights}

If we analyze equation (2) more carefully, we can observe that most weights appear only in the products of weights and can be arbitrary when those products evaluate to zero. Moreover, weights of the format $\alpha_{i}\left(\emptyset,{ }_{--}\right)$appear only in marginal contributions of the form $\left[m c_{i}^{\alpha}(v)\right](\{i\}, P)$. These marginal contributions always evaluate to $v(\{i\}, P)$ and are independent of weights. This leads us to the notion of significance.

- Significant weights: weight $\alpha_{i}(S, P)$ is called significant if $(S, P)$ is nonempty and probable, that is, if $S \neq \emptyset$ and $\sum_{\pi \in \Omega(N \backslash(S \cup\{i\}))} \operatorname{pr}_{\pi}^{\alpha}\left(S_{+i}, \tau_{i}^{S}(P)\right) \neq$ 0 .

If we limit ourselves to non-negative weights, then $\alpha_{i}(S, P)$, such that $S \neq \emptyset$, is significant if and only if there exists a permutation $\pi$ such that $p_{\pi}^{\alpha}(S, P)>0$. In turn, if all weights are positive, then all $\alpha_{i}(S, P)$ such that $S \neq \emptyset$ are significant.

The following lemma states that only significant weights have an impact on the value.

Lemma 1 Let $\alpha$-marginality and $\hat{\alpha}$-marginality be two definitions of marginal contribution. Then, $\alpha$-value and $\hat{\alpha}$-value differs if and only if there exists an embedded coalition $(S, P)$ such that $\alpha(S, P) \neq \hat{\alpha}(S, P)$ and both weights are significant.

Proof. First, we will prove that insignificant weights do not change the value. Based on equation (2), weight $\alpha_{i}(\emptyset, P)$ appears only in the marginal contribution $\left[m c_{i}(v)\right]\left(\{i\}, \tau_{i}^{\emptyset}(P)\right)=\sum_{T \in P} \alpha_{i}\left(\emptyset, \tau_{i}^{T}(P)\right)\left[v(\{i\}, P)-v\left(\emptyset, \tau_{i}^{T}(P)\right)\right]=v(\{i\}, P)$ (therefore the particular weights do not matter as long as they sum up to one). Now, let us consider in which place insignificant weight $\alpha_{i}(S, P)$ with $S \neq \emptyset$ appears in equation (2). When we calculate the payoff of player $i$, weight $\alpha_{i}(S, P)$ appears only in marginal contribution $\left[m c_{i}(v)\right]\left(S_{+i}, \tau_{i}^{S}(P)\right)$ preceded by $\sum_{\pi \in \Omega(N \backslash(S \cup\{i\}))} \operatorname{pr}_{\pi}^{\alpha}\left(S_{+i}, \tau_{i}^{S}(P)\right)$ which equals zero. In the payoff of other players, $\alpha_{i}(S, P)$ appears in the sum $\sum_{\tilde{\pi} \in N \backslash \tilde{S}} p r_{\tilde{\pi}}^{\alpha}(\tilde{S}, \tilde{P})$, where a given product 
is obtained by the sequence of transfers that transform $(N,\{N, \emptyset\})$ into $(\tilde{S}, \tilde{P})$. Thus, $\alpha_{i}(S, P)$ appears only if all other players from $N \backslash S$ have transferred before $i$ (and only them):

$$
\sum_{\tilde{\pi} \in N \backslash \tilde{S}} r_{\tilde{\pi}}^{\alpha}(\tilde{S}, \tilde{P})=\sum_{\pi_{2} \in \Omega(S \backslash \tilde{S})} \sum_{\pi_{1} \in \Omega(N \backslash(S \cup\{i\}))} p r_{\pi_{1}|| i|| \pi_{2}}^{\alpha}(\tilde{S}, \tilde{P}) .
$$

Now, for a given permutation of players from $S \backslash \tilde{S}: \pi_{2} \in \Omega(S \backslash \tilde{S})$, if we extract the product of last $|S|-|\tilde{S}|$ weights from the second sum, we see that the whole sum is multiplied by $\sum_{\pi \in \Omega(N \backslash(S \cup\{i\}))} \operatorname{pr}_{\pi}^{\alpha}\left(S_{+i}, \tau_{i}^{S}(P)\right) \cdot \alpha_{i}(S, P)$. This equals zero regardless of weight $\alpha_{i}(S, P)$.

Now, we will prove that the significant weight has an impact on the value. Assume that $\alpha_{1}$ and $\alpha_{2}$ differ in at least one significant weight. Let $(S, P)$ be an embedded coalition such that $\alpha_{i}(S, P) \neq \hat{\alpha}_{i}(S, P)$, both weights are significant and $\alpha_{i}(\tilde{S}, \tilde{P})=\hat{\alpha}_{i}(\tilde{S}, \tilde{P})$ for every $(\tilde{S}, \tilde{P})$ with $S \subset \tilde{S}$. Now, consider simple game $e^{(S, P)}$. As $p r_{\pi}^{\alpha_{1}}\left(S_{+i}, \tau_{i}^{S}(P)\right)=p r_{\pi}^{\alpha_{2}}\left(S_{+i}, \tau_{i}^{S}(P)\right)$ for every $\pi$ and, from weights significance, we have that $\sum_{\pi \in \Omega(N \backslash(S \cup\{i\}))} p r_{\pi}^{\alpha}\left(S_{+i}, \tau_{i}^{S}(P)\right) \neq 0$, then from equation (2) we get $\varphi_{i}^{\alpha}\left(v^{(S, P)}\right) \neq \varphi_{i}^{\hat{\alpha}}\left(v^{(S, P)}\right)$.

A direct consequence of the above lemma is that we will focus only on significant weights.

We conclude this section by discussing how weights associated with elementary marginal contributions can be looked upon as weights of partitions. To this end, let $(S, P) \in E C$ such that $i \notin S$ and let $\alpha_{i}(S, P)$ be significant. Then, there exists $\pi \in \Omega(N \backslash(S \cup\{i\}))$ such that $\operatorname{pr}_{\pi}^{\alpha}\left(S_{+i}, \tau_{i}^{S}(P)\right) \neq 0$. Now, we have that:

$$
\alpha_{i}(S, P)=\frac{p r_{\pi \| i}^{\alpha}(S, P)}{p r_{\pi}^{\alpha}\left(S_{+i}, \tau_{i}^{S}(P)\right)},
$$

that is, all significant weights $\alpha$ can be calculated from products $p r^{\alpha}$. Moreover, if we use equation (5) for different embedded coalitions obtained by the transfer of $i$ from $\left(S_{+i}, \tau_{i}^{S}(P)\right)$, we get $p r_{\pi}^{\alpha}\left(S_{+i}, \tau_{i}^{S}(P)\right)=\sum_{T \in P_{-S}} p r_{\pi \| i}^{\alpha}\left(S, \tau_{i}^{T}(P)\right)$ (i.e., all products $p r^{\alpha}$ for bigger coalitions can be obtained from products for smaller coalitions). That leads us to an observation that defining values of $\operatorname{pr}_{\pi}^{\alpha}(\emptyset, P)$ for every $\pi \in \Omega(N)$ and every partition $P$ is equivalent to defining significant weights $\alpha$. Based on the conditions imposed on weights $\alpha$, all weights compositions must sum up to one (i.e., $\sum_{P \in \mathcal{P}} \operatorname{pr}_{\pi}^{\alpha}(\emptyset, P)=1$ for every $\pi \in \Omega(N)$ ) and must be symmetrical (i.e., $p r_{\pi_{1}}^{\alpha}\left(\emptyset, \pi_{1}(P)\right)=p r_{\pi_{2}}^{\alpha}\left(\emptyset, \pi_{2}(P)\right)$ for every $\pi \in \Omega(N)$ and $P \in$ $\mathcal{P})$.

This observation, seemingly trivial, allows us to focus on the weights of partitions (that may represent the probability that a given partition will form) instead of considering elementary transfers. To give an example, Hu and Yang argued that, independently of a permutation of players, all partitions should be equally likely to form [9]. Thus, $p_{\pi}^{\alpha^{H Y}}(\emptyset, P)=\frac{1}{\mathcal{P}(N)}$ for every $\pi \in \Omega(N)$ and $P \in \mathcal{P}(N)$. 
That immediately implies weights $\alpha: \operatorname{pr}_{\pi}^{\alpha^{H Y}}(S, P)=\frac{\left|\left\{R \in \mathcal{P}(N): R_{[S\}}=P_{[S]}\right\}\right|}{|\mathcal{P}(N)|}$ and $\alpha_{i}^{H Y}(S, P)=\frac{\left|\left\{R \in \mathcal{P}(N): R_{[S]}=P_{[S\}}\right\}\right|}{\mid\left\{R \in \mathcal{P}(N): R_{[S \cup\{i\}]}=P_{[S \cup\{i\}]} \mid\right.}$.

\subsection{Weak and Strong Monotonicity}

One of the desirable properties of the division scheme is that, if we increase the value of a particular coalition, then the payoffs, that is the shares of the grand coalition value assigned to its members, should not decrease. By the same token, the shares of non-members should not increase. This property is called (Weak) Monotonicity. Indeed, the fact that Myerson's value violates Monotonicity is the main reason why it was criticized in the literature as unintuitive $[10,3,11]$. Formally, we have the following definition:

- Weak Monotonicity (increase of player's contributions does not decrease its payoff): if $v_{1}\left(S_{+i}, \tau_{i}^{S}(P)\right)-v_{1}(S, P) \geq v_{2}\left(S_{+i}, \tau_{i}^{S}(P)\right)-v_{2}(S, P)$ holds for every $(S, P) \in E C$, such that $i \notin S$, then $\varphi_{i}\left(v_{1}\right) \geq \varphi_{i}\left(v_{2}\right)$.

This formulation agrees with definitions proposed by Macho-Stadler et al. and De Clippel and Serrano, and differs from the one by McQuillin. ${ }^{10}$

Now, we prove that the necessary and sufficient condition for the Weak Monotonicity to be met by $\alpha$-value is that weights $\alpha$ are non-negative.

Lemma $2 \alpha$-value satisfies Weak Monotonicity if and only if $\alpha_{i}(S, P) \geq 0$ for every significant weight.

Proof. First, let us transform one more time the formula for $\alpha$-value:

(6) $\quad \varphi_{i}^{\alpha}(v)=\sum_{(S, P) \in E C, i \notin S} \frac{|S| !}{|N| !} \sum_{\pi \in \Omega\left(N \backslash S_{+i}\right)} p r_{\pi|| i}^{\alpha}(S, P) \cdot\left[v\left(S_{+i}, \tau_{i}^{S}(P)\right)-v(S, P)\right]$.

In the formula for $\varphi_{i}^{\alpha}$ the coefficient of the marginal contribution $v\left(S_{+i}, \tau_{i}^{S}(P)\right)-$ $v(S, P)$ equals $\frac{|S| !}{|N| !} \sum_{\pi \in \Omega\left(N \backslash S_{+i}\right)} p r_{\pi|| i}^{\alpha}(S, P)$ which is equivalent to

$$
\frac{|S| !}{|N| !} \sum_{\pi \in \Omega\left(N \backslash S_{+i}\right)} \operatorname{pr}_{\pi}^{\alpha}\left(S_{+i}, \tau_{i}^{S}(P)\right) \cdot \alpha_{i}(S, P) .
$$

If this coefficient is negative, then its increase will decrease the payoff. Thus, $\alpha$ value satisfies Weak Monotonicity if and only if all coefficients are non-negative. If all weights are non-negative then all products of weights are non-negative

\footnotetext{
${ }^{10} \mathrm{McQuillin}$ in his definition required only that the increase of a coalition's value causes no decrease of payoffs of the members. Formally, he defined Weak Monotonicity as follows: $\varphi_{i}\left(e^{(S, P)}\right) \geq 0$ if $i \in S$. For linear values, this definition is equivalent to the following one: if $v_{1}(S, P) \geq v_{2}(S, P)$ holds for every $(S, P) \in E C$, such that $i \in S$, then $\varphi_{i}\left(v_{1}\right) \geq \varphi_{i}\left(v_{2}\right)$. Indeed, in games with no externalities, this implies for symmetric values that other players' payoffs do not increase. However, this is not the case when externalities are present. For example, in a simple game $e^{(S, P)}$ for $(S, P)=(\{1\},\{\{1\},\{2\},\{3,4\}\})$ the following payoff scheme $\varphi_{1}\left(e^{(S, P)}\right)=\varphi_{2}\left(e^{(S, P)}\right)=a$ and $\varphi_{3}\left(e^{(S, P)}\right)=\varphi_{4}\left(e^{(S, P)}\right)=-a$ does not violate Symmetry, nor Weak Monotonicity.
} 
(thus, the condition is satisfied). In turn, using the reverse induction on $|S|$, we get that all significant weights $\alpha_{i}(S, P)$ must be non-negative: from inductive assumption, $\operatorname{pr}_{\pi}^{\alpha}\left(S_{+i}, \tau_{i}^{S}(P)\right) \geq 0$ for every $\pi \in \Omega\left(N \backslash S_{+i}\right)$, thus if the sum is greater than zero, then $(S, P)$ is probable and must be non-negative. This concludes our proof.

In Weak Monotonicity, we require that the increase of a player's contribution does not result in the decrease of their payoff. This, in particular, means that we allow for a hypothetical situation where a player's arbitrary big contributions to some coalitions, although not negatively affecting his payoff, may not affect his payoff at all. This would be discouraging for players. To address this issue, we propose a notion of Strong Monotonicity:

- Strong Monotonicity (increase of player's contributions increases its payoff): if $v_{1}\left(S_{+i}, \tau_{i}^{S}(P)\right)-v_{1}(S, P) \geq v_{2}\left(S_{+i}, \tau_{i}^{S}(P)\right)-v_{2}(S, P)$ holds for every $(S, P) \in E C$, such that $i \notin S$ and this inequality is strict for at least one embedded coalition, then $\varphi_{i}\left(v_{1}\right)>\varphi_{i}\left(v_{2}\right)$.

Lemma $3 \alpha$-value satisfies Strong Monotonicity if and only if $\alpha_{i}(S, P)>0$ for every significant weight.

Proof. Proof is analogous to the proof of Lemma 2, but here all coefficients must be positive (otherwise, if $v\left(S_{+i}, \tau_{i}^{S}(P)\right)-v(S, P)$ has zero coefficients, then the payoff in game $-e^{(S, P)}$ does not increase the payoff for player $\left.i\right)$.

The above lemma shows that Strong Monotonicity implies that, in the stochastic process (which is built upon the $\alpha$ value), every transfer is possible.

Next, we will analyze the axiom of Strong Symmetry.

\subsection{Strong Symmetry}

Macho-Stadler et al. proposed a strengthening of the axiom of Symmetry called Strong Symmetry. To look closer into this concept, let us consider simple game $e^{(S, P)}$ (where only particular $S$ embedded in $P$ has non-zero value). From Symmetry, all players from $S$ have the same payoff. In turn, payoffs of players from $N \backslash S$ may differ between them. This may seem unfair, as they all have the same role in this game: they must form specific partition $P$ for $S$ to generate a value.

Let us then consider a bijection (i.e., one-to-one mapping) $f: N_{-S} \rightarrow N_{-S}$. The axiom of Strong Symmetry says that, if $f_{(S, P)}(v)$ is a game obtained by exchanging the value of $(S, P)$ and $(S, S \cup f(P \backslash S)),{ }^{11}$ then all the payoffs from game $f_{(S, P)}(v)$ are the same as the payoffs from game $v$ :

- Strong Symmetry (the value of a coalition affects the payoffs of outside players symmetrically):

\footnotetext{
${ }^{11}$ Formally, $\left[f_{(S, P)}(v)\right](S, P)=v(S, S \cup f(P \backslash S)),\left[f_{(S, P)}(v)\right](S, S \cup f(P \backslash S))=v(S, P)$ and $\left[f_{(S, P)}(v)\right](\tilde{S}, \tilde{P})=v(\tilde{S}, \tilde{P})$, otherwise. See Symmetry definition for a formal specification of $f(P)$.
} 
- $\varphi(f(v))=f(\varphi)(v)$ for every game $v$ and bijection $f \in \Omega(N)$;

- $\varphi\left(f_{(S, P)}(v)\right)=\varphi(v)$ for every game $v$ and bijection $f \in \Omega(N \backslash S)$;

This definition is equivalent to the condition $\varphi_{i}\left(e^{(S, P)}\right)=\varphi_{j}\left(e^{(S, P)}\right)$ for every $i, j \notin S$ for linear values.

To translate this axiom to a property of the weight, we introduce the concept of the interlace resistance.

- Interlace resistance (product of weights should not depend on the order of corresponding transfers):

$$
p r_{\pi_{1}}^{\alpha}(S, P)=p r_{\pi_{2}}^{\alpha}(S, P)
$$

for every $(S, P)$ such that $S \neq \emptyset$ and every $\pi_{1}, \pi_{2} \in \Omega\left(N_{-S}\right)$.

For non-negative weights this condition simplifies to the equivalence of products of two weights.

Lemma 4 If weights $\alpha$ are non-negative, then $\alpha$ is interlace resistance if and only if it satisfies $\alpha_{i}(S, P) \cdot \alpha_{j}\left(S_{+i}, \tau_{i}^{S}(P)\right)=\alpha_{j}(S, P) \cdot \alpha_{i}\left(S_{+j}, \tau_{j}^{S}(P)\right)$ for all significant weights such that $i, j \notin S$.

Proof. First, we prove that the above condition is necessary. Let $(S, P)$ be an embedded coalition such that $i, j \notin S, S \neq \emptyset$ and $(S, P)$ is probable (note that, for non-negative weights, if $(S, P)$ is probable then $\left(S_{+i}, \tau_{i}^{S}(P)\right),\left(S_{+j}, \tau_{j}^{S}(P)\right)$ and $\left(S_{+i j}, \tau_{j}^{S}\left(\tau_{i}^{S}(P)\right)\right)$ are also probable). Let $\pi$ be a permutation of $N \backslash(S \cup\{i, j\})$ such that $p r_{\pi}\left(S_{+i j}, \tau_{i j}^{S}(P)\right)>0$. Now, let us consider two different extensions of permutation $\pi: \pi\|j\| i, \pi\|i\| j \in \Omega\left(N_{-S}\right)$ (i.e., the first one ends with $(j, i)$, and the second one with $(i, j))$. Now, the condition $p r_{\pi\|j\| i}(S, P)=p r_{\pi\|i\| j}(S, P)$ simplifies to $\alpha_{i}(S, P) \cdot \alpha_{j}\left(S_{+i}, \tau_{i}^{S}(P)\right)=\alpha_{j}(S, P) \cdot \alpha_{i}\left(S_{+j}, \tau_{j}^{S}(P)\right)$.

Next, we show that this condition is sufficient. This comes from the combinatorial fact that every permutation can be obtained from another using only transpositions of the adjacent elements. More formally, assume that $(S, P)$ is an embedded coalition with $S \neq \emptyset$ and $\pi_{1}, \pi_{2}$ are some permutations of $N_{-S}$. We will prove that both products of weights $p r_{\pi_{1}}^{\alpha}(S, P), p r_{\pi_{2}}^{\alpha}(S, P)$ are equal if the condition holds. To this end, we observe that if in both products a zero weight appears then both are equal. If not, without the loss of generality, all weights in $\operatorname{pr}_{\pi_{1}}^{\alpha}(S, P)$ are nonzero and significant. Based on the condition $\alpha_{i}(S, P) \cdot \alpha_{j}\left(S_{+i}, \tau_{i}^{S}(P)\right)=$ $\alpha_{j}(S, P) \cdot \alpha_{i}\left(S_{+j}, \tau_{j}^{S}(P)\right)$, transpositions of adjacent players in order $\pi_{1}$ do not change the product of weights. Thus, the proper sequence of transpositions will yield that $p r_{\pi_{1}}(S, P)=p r_{\pi_{2}}(S, P)$.

The following theorem shows that an $\alpha$-value satisfies Strong Symmetry if and only if weights are interlace resistant.

Theorem 3 An $\alpha$-value satisfies Strong Symmetry if and only if $\alpha$-marginality is interlace resistant.

Proof. Assume that $\alpha$ is interlace resistant. We will prove that for every $(S, P)$ and $i, j \in N \backslash S$ holds $\varphi_{i}\left(e^{(S, P)}\right)=\varphi_{j}\left(e^{(S, P)}\right)$. This fact, based on Additivity, 
will imply Strong Symmetry. Let $\pi_{e} \in \Omega(N \backslash S)$ be any permutation. Based on equation (6):

$$
\varphi_{i}^{\alpha}\left(e^{(S, P)}\right)=-\frac{|S| !(|N|-|S|+1) !}{|N| !} \cdot \operatorname{pr}_{\pi_{e}}^{\alpha}(S, P)
$$

do not depend on the player $i \notin S$. Thus, $\varphi_{i}\left(e^{(S, P)}\right)=\varphi_{j}\left(e^{(S, P)}\right)$.

To prove that Strong Symmetry holds only for interlace resistant weights we use the reverse induction on the size of $S$. Of course $p r_{\pi_{1}}(S, P)=p r_{\pi_{2}}(S, P)=1$ when $|S|=|N|-1$ for every permutation $\pi_{1}, \pi_{2} \in \Omega\left(N_{-S}\right)$. Let us assume that this equivalence holds when $|S|>k$. We will prove that it also holds when $|S|=k$. Let $\pi_{1}, \pi_{2} \in \Omega(N \backslash S)$ be two permutations, and without the loss of generality assume $i$ and $j$ are the last players in $\pi_{1}, \pi_{2}$. Now, consider simple game $e^{(S, P)}$ in which $(S, P)$ is the only embedded coalition with non-zero value. Based on Strong Symmetry, players $i$ and $j$ have equal payoffs: $\varphi_{i}^{\alpha}\left(e^{(S, P)}\right)=\varphi_{j}^{\alpha}\left(e^{(S, P)}\right)$. From equation (6):

$$
\varphi_{i}^{\alpha}\left(e^{(S, P)}\right)=-\frac{|S| !}{|N| !} \sum_{\pi \in \Omega\left(N \backslash S_{+i}\right)} p r_{\pi}^{\alpha}\left(S_{+i}, \tau_{i}^{S}(P)\right) \cdot \alpha_{i}(S, P) .
$$

Based on the inductive assumption, product $\operatorname{pr}_{\pi}^{\alpha}\left(S_{+i}, \tau_{i}^{S}(P)\right)$ does not depend on the permutation $\pi \in \Omega\left(N \backslash S_{-i}\right)$, thus $\varphi_{i}^{\alpha}\left(e^{(S, P)}\right)=-\frac{(|S| !)(|N|-|S|+1) !}{|N| !} p r_{\pi_{1}}^{\alpha}(S, P)$. Now, $\varphi_{j}^{\alpha}\left(e^{(S, P)}\right)=-\frac{(|S| !)(|N|-|S|+1) !}{|N| !} p r_{\pi_{2}}^{\alpha}(S, P)$ implies $p r_{\pi_{1}}^{\alpha}(S, P)=p r_{\pi_{2}}^{\alpha}(S, P)$.

When we consider the values that satisfy Strong Symmetry, the general formula (2) can be simplified as follows:

$$
\varphi_{i}^{\alpha}(v)=\sum_{(S, P) \in E C, i \in S} \frac{(|S|-1) !(|N|-|S|) !}{|N| !} \operatorname{pr}^{\alpha}(S, P)\left[m c_{i}(v)\right](S, P),
$$

where $\operatorname{pr}^{\alpha}(S, P)$ denotes $\operatorname{pr}_{\pi}^{\alpha}(S, P)$ for any permutation $\pi \in \Omega(N \backslash S)$, as this product is equal for every permutation. Observe that $v(S, P)$ appears in the above formula multiplied by $\mathrm{pr}^{\alpha}(S, P)$ and $v\left(S_{-i}, \tau_{i}^{T}(P)\right)$ - $\operatorname{by~} r^{\alpha}(S, P) \cdot \alpha_{i}\left(S_{-i}, \tau_{i}^{T}(P)\right)=$ $\operatorname{pr}^{\alpha}\left(S_{-i}, \tau_{i}^{T}(P)\right)$. Thus, the value of a coalition in a given partition is always preceded with the probability that a given partition will form. This suggests the following algorithm for evaluating a fair division in a game with externalities:

- first, we create average game $\tilde{v}$ with no externalities from game $v$ with externalities. This is done by calculating the value of every coalition as the average of its values in games with externalities:

$$
\tilde{v}(S)=\sum_{P \ni S} a(S, P) \cdot v(S, P),
$$

where $\sum_{P \ni S} a(S, P)=1$ for every $S$; 
- then, we calculate the Shapley value for average game $\tilde{v}$ :

$$
\varphi(v)=S h(\tilde{v}) .
$$

This approach, called the average approach, was introduced by Macho-Stadler et al. They proved that the value that satisfies Shapley's axioms can be constructed using the average approach if and only if it satisfies Strong Symmetry (see Theorem 1 in [10]).

In our Theorem 2 we proved that marginality approach can produce every value that satisfies Shapley's axiom and Theorem 3 shows that the resulting value satisfies Strong Symmetry if and only if weights are interlace resistant. Thus, our two theorems combined with the result from Macho-Stadler et al. imply that a value can be obtained using the average approach if and only if it can be obtained using the marginality approach with interlace resistant weights. Corollary 1 Average approach is equivalent to marginality approach with interlace resistant weights.

Finally, in the next section we consider the Strong Null-Player Axiom.

\subsection{Strong Null-Player Axiom}

Another axiom proposed by Macho-Stadler et al. is the Strong Null-Player Axiom. Let us consider game $v$ in which $i$ is a null-player in a strict sense, i.e., $i$ does not have an impact on the game whatsoever. In this case, the Null-Player Axiom requires that player $i$ has zero payoff: $\varphi_{i}(v)=0$. But it does not mean that he has no impact on others' payoffs. In other words, removing a null-player from the game may affect payoffs of the remaining players. Such a situation is infeasible if we rely on the Strong Null-Player Axiom proposed by Macho-Stadler et al.:

- Strong Null-Player Axiom (null-player does not have an impact on the payoffs of others): if $i$ is a null-player then $\varphi_{j}(v)=\varphi_{j}\left(v_{-i}\right)$ for every $j \in N$, where $v_{-i}$ denotes the game without player $i: v_{-i}\left(S_{-i}, P_{-i}\right) \stackrel{\text { def }}{=} v(S, P)$ for every $(S, P)$ such that $i \in S$.

When $i$ is not a null-player, constructing game $v_{-i}$ can be challenging. This issue will be discussed in more detail in Section 5. However, the situation is much simpler when $i$ is a null-player. This is because the value of embedded coalition $(S, P) \in E C(N \backslash\{i\})$ can be obtained by inserting $i$ to an arbitrary coalition in partition $P$. No matter where $i$ was, it would not impact the value of $(S, P)$.

Let us now analyze the constraints imposed by the Strong Null-Player Axiom on the values that satisfy Strong Symmetry. To this end, let us introduce the expansion resistance property.

- Expansion resistance (weight does not depend on the size of $S$ ):

$$
\alpha_{i}(S, P)=\alpha_{i}\left(S_{-j}, P_{-j}\right)
$$

for all significant weights such that $i \notin S$ and $j \in S$. 
In terms of our bargaining process, this intuitive requirement says that the probability of joining a coalition by a player should depend only on the coalitions to choose from and not on the coalition that the player is leaving. The following theorem states that, for values satisfying Strong Symmetry, expansion resistance is necessary and sufficient to obtain the Strong Null-Player Axiom.

Theorem 4 Let $\alpha$-value satisfy Strong Symmetry. Then, it satisfies the Strong Null-Player Axiom if and only if $\alpha$-marginality is expansion resistant.

Proof. In this proof we consider only $\alpha$-values that satisfy Strong Symmetry. We will show that expansion resistance is equivalent to satisfying the Strong Null-Player Axiom by the sequence of equivalences.

PART 1: expansion resistance $\Leftrightarrow p r^{\alpha}(S, P)=p r^{\alpha}\left(S_{-i}, P_{-i}\right)$ for every $(S, P)$ such that $i \in S$;

It is clear that expansion resistance implies the condition from the right-hand side. Also, if expansion resistance is not met, then products of weights must also differ: if $(S, P)$ is the smallest coalition such that $\alpha_{j}(S, P) \neq \alpha_{j}\left(S_{-i}, P_{-i}\right)$ then $p r^{\alpha}\left(S_{+j}, \tau_{j}^{S}(P)\right)=p r^{\alpha}\left(\left(S_{-i}\right)_{+j}, \tau_{j}^{S}\left(P_{-i}\right)\right)$ and $p r^{\alpha}(S, P) \neq p r^{\alpha}\left(S_{-i}, P_{-i}\right)$.

PART 2: $p r^{\alpha}(S, P)=p r^{\alpha}\left(S_{-i}, P_{-i}\right) \Leftrightarrow \varphi_{j}^{\alpha}\left(e^{(S, P)}\right)=\frac{|S|-1}{|N|} \cdot \varphi_{j}^{\alpha}\left(e^{\left(S_{-i}, P_{-i}\right)}\right)$ for $i, j \in$ $S$

This step, which translate weights characteristic to the value property is immediate from equation (8). Now, let us denote $\tilde{v}^{(S, P)}=e^{(S, P)}+\sum_{T \in P_{-S}} e^{\left(S_{-i}, \tau_{i}^{T}(P)\right)}$.

Thus, $\tilde{v}_{-i}^{(S, P)}=e^{\left(S_{-i}, P_{-i}\right)}$.

PART 3: $\varphi_{j}^{\alpha}\left(e^{(S, P)}\right)=\frac{|S|-1}{|N|} \cdot \varphi_{j}^{\alpha}\left(e^{\left(S_{-i}, P_{-i}\right)}\right)$ for $i, j \in S \Leftrightarrow \varphi_{j}^{\alpha}\left(\tilde{v}^{(S, P)}\right)=\varphi_{j}^{\alpha}\left(\tilde{v}_{-i}^{(S, P)}\right)$ for $j \in$ $S$ and null-player $i \in S$

The right-hand side of the equivalence comes from the Strong Null-Player Axiom applied to game $\tilde{v}^{(S, P)}$. To prove equivalence, we will transform it using Additivity:

$$
\varphi_{j}^{\alpha}\left(e^{(S, P)}\right)+\sum_{T \in P_{-S}} \varphi_{j}^{\alpha}\left(e^{\left(S_{-i}, \tau_{i}^{T}(P)\right)}\right)=\varphi_{j}^{\alpha}\left(e^{\left(S_{-i}, P_{-i}\right)}\right) .
$$

But based on Strong Symmetry all payoffs of players outside $S_{-i}$ in $e^{\left(S_{-i}, \tau_{i}^{T}(P)\right)}$ are equal, thus from Efficiency $\varphi_{j}\left(e^{\left(S_{-i}, \tau_{i}^{T}(P)\right)}\right) \cdot(|S|-1)=-\varphi_{i}\left(e^{\left(S_{-i}, \tau_{i}^{T}(P)\right)}\right) \cdot$ $(|N|-|S|+1)$ for every $T \in P_{-S}$. Thus,

$$
\begin{aligned}
\varphi_{j}\left(e^{\left(S_{-i}, P_{-i}\right)}\right) & =\varphi_{j}\left(e^{(S, P)}\right)-\frac{|N|-|S|+1}{|S|-1} \sum_{T \in P_{-S}} \varphi_{i}\left(e^{\left(S_{-i}, \tau_{i}^{T}(P)\right)}\right) \\
& =\varphi_{j}\left(e^{(S, P)}\right)+\frac{|N|-|S|+1}{|S|-1} \cdot \varphi_{i}\left(e^{(S, P)}\right)=\frac{|N|}{|S|-1} \cdot \varphi_{j}\left(e^{(S, P)}\right),
\end{aligned}
$$

where we used Symmetry $\varphi_{i}\left(e^{(S, P)}\right)=\varphi_{j}\left(e^{(S, P)}\right)$ and the Null-Player Axiom for equality $\sum_{T \in P_{-S}} \varphi_{i}\left(e^{\left(S_{-i}, \tau_{i}^{T}(P)\right)}\right)=-\varphi_{i}\left(e^{(S, P)}\right)$.

PART 4: $\varphi_{j}^{\alpha}\left(\tilde{v}^{(S, P)}\right)=\varphi_{j}^{\alpha}\left(\tilde{v}_{-i}^{(S, P)}\right)$ for every $(S, P)$ such that $j \in S$ and null-player $i \in$ $S \Leftrightarrow$ Strong Null-Player Axiom 
Of course the Strong Null-Player Axiom implies the left-hand side. On the other hand, every game in which $i$ is a null-player can be decomposed in the following way:

$$
v=\sum_{(S, P), i \in S} v(S, P) \cdot\left(e^{(S, P)}+\sum_{T \in P_{-S}} e^{\left(S_{-i}, \tau_{i}^{T}(P)\right)}\right)=\sum_{(S, P), i \in S} v(S, P) \cdot \tilde{v}^{(S, P)} .
$$

Thus, again based on Additivity, if value $\varphi_{j}^{\alpha}\left(\tilde{v}^{(S, P)}\right)=\varphi_{j}^{\alpha}\left(\tilde{v}_{-i}^{(S, P)}\right)$ holds for every $(S, P)$ and $j \in S$, then $\varphi_{j}^{\alpha}(v)=\varphi_{j}^{\alpha}\left(v_{-i}\right)$. That implies also $\varphi_{j}^{\alpha}(v)=\varphi_{j}^{\alpha}\left(v_{-i}\right)$ for $j \notin S$ from Strong Symmetry and concludes our proof.

\section{RELATIONSHIP WITH YOUNG'S AND MYERSON'S AXIOMATIZATIONS}

In this section we will discuss how both Young's Monotonicity axiomatization and Myerson's axiomatization based on the concept of balanced contribution can be translated to games with externalities.

Young argues that the concept of (Weak) Monotonicity can be restated as follows: if we consider two games such that in the first game all marginal contributions of a player are not smaller than the corresponding marginal contributions in the second game (i.e., the difference of vectors of marginal contributions is non-negative in every coordinate), then the payoff in the former game should not be smaller than the payoff in the latter game. This yields another property, called the Marginality Axiom - this says that if marginal contributions are equal, then the payoffs should also be equal. In other words, payoffs should depend only on the vector of marginal contributions. Young proved that the Shapley value is the only value which satisfies Efficiency, Symmetry and Marginality.

In games with externalities, we have to specify which definition of marginal contribution we assume. That leads to the $\alpha$-Marginality Axiom:

- $\alpha$-Marginality Axiom (payoff of a player depends only on his marginal contributions): $m c_{i}^{\alpha}\left(v_{1}\right)=m c_{i}^{\alpha}\left(v_{2}\right) \Rightarrow \varphi_{i}\left(v_{1}\right)=\varphi_{i}\left(v_{2}\right)$ for every game $v_{1}, v_{2}$ and agent $i \in N$.

Bolger [2] used Young's axiomatization to derive his value (with an additional Null-Player Axiom which is, in fact, redundant). Later, De Clippel and Serrano proved that externality-free value proposed by Pham Do and Norde (initially introduced using Shapley's standard axiomatization) can be also derived using this set of axioms [3]. Finally, Fujinaka provided a general theorem: for every definition of marginal contribution there exists a unique value which satisfies the Efficiency, Symmetry and $\alpha$-Marginality Axioms [6]. For every $\alpha$, the value proposed by Fujinaka based on Young's axiomatization is equal to our value (derived by Theorem 1). This means that both axiomatizations are equivalent. Corollary 2 Shapley's marginality-based axiomatization (Efficiency, Symmetry, Additivity and $\alpha$-Null-Player Axiom) is equivalent to Young's axiomatization (Efficiency, Symmetry and $\alpha$-Marginality Axiom). Moreover, both axiomatizations yield a unique value. 
Next, we will discuss an axiomatization proposed by Myerson [13] that is based on the concept of Balanced Contributions. We translate this axiom to games with externalities using our analysis of marginal contributions. It comes out that not every value obtained using the marginality approach satisfies the axiom of Balanced Contributions. Therefore, we characterize which values satisfy Myerson's concept using the properties of interlace and expansion resistance.

The Balanced Contributions principle guarantees a certain notion of stability. We say that mutual contributions of players $i$ and $j$ are balanced, if the withdrawal of player $i$ from the game will result in the same loss to player $j$, as the withdrawal of $j$ to $i$. More formally, $\varphi_{i}(v)-\varphi_{i}\left(v_{-j}\right)=\varphi_{j}(v)-\varphi_{j}\left(v_{-i}\right)$. Thus, the profit of cooperation is divided equally between players. It is important that this condition is met, as otherwise a player which gains less may threaten the other to leave the game. This is why the Balanced Contributions principle is usually the key piece in the mechanisms that implement the Shapley value (see, e.g., Perez-Castrillo et al. [15]).

To translate this axiom to games with externalities we need to define how to calculate a game without a given player. In games without externalities, the value of a coalition $S$ without a player $i$ is uniquely defined. In games with externalities, different positions of $i$ in the coalition structure may result in different values of $S$. Let us consider the following example:

Example 3 Let us consider game $v_{-1}$ created from game $v$ in Example 1. We have that $v_{-1}(\{2\},\{\{2\},\{3\}, \emptyset\})=b$ if we take value of $\{2\}$ from $\{\{1\},\{2\},\{3\}, \emptyset\}$ and $v_{-1}(\{2\},\{\{2\},\{3\}, \emptyset\})=c$ if we take value from $\{\{1,3\},\{2\}, \emptyset\}$.

This resembles the problem with defining the marginal contribution we faced before. Thus, we take a similar approach: from the value of coalition $S \cup\{i\}$, we subtract player's $i$ marginal contribution:

$$
v_{-i}^{\alpha}\left(S_{-i}, P_{-i}\right) \stackrel{\text { def }}{=} v(S, P)-m c_{i}^{\alpha}(S, P)
$$

for every $(S, P)$ such that $i \in S .^{12}$ Now, different definitions of marginal contributions $(i . e$. weights $\alpha$ ) result in different values of the game without a player. Therefore, the corresponding axioms of Balanced Contributions will also be different:

- $\alpha$-Balanced Contributions (profit of cooperation is divided equally between players): $\varphi_{i}(v)-\varphi_{i}\left(v_{-j}^{\alpha}\right)=\varphi_{j}(v)-\varphi_{j}\left(v_{-i}^{\alpha}\right)$ for every game $v$ and $i, j \in N$.

The principle of Balanced Contributions combined with Efficiency automatically yields a recursive formula for the unique value:

$$
\varphi_{i}(v)=\frac{1}{|N|}\left(v(N,\{N, \emptyset\})-v\left(\left\{N_{-i},\left\{N_{-i},\{i\}, \emptyset\right\}\right)+\sum_{j \neq i} \varphi_{i}\left(v_{-j}^{\alpha}\right)\right) .\right.
$$

This comes from the sum of Balanced Contributions equations over all $j \in N$.

\footnotetext{
${ }^{12}$ It is worth noting that under this definition of the game without a player $\alpha$-values that satisfy Strong Symmetry and the Strong Null-Player Axiom satisfy also the Strong $\alpha$-NullPlayer Axiom that states that if $i$ is an $\alpha$-null-player then $\varphi_{j}(v)=\varphi_{j}\left(v_{-i}^{\alpha}\right)$.
} 
For games without externalities, Myerson proved that Efficiency and Balanced Contributions imply the Shapley value (thus it is equivalent, in particular, to Shapley's and Young's axiomatizations). But as it appears, this is not the case in games with externalities - some $\alpha$-values do not meet the corresponding axiom of Balanced Contributions parametrized with $\alpha$.

To characterize which values meet Balanced Contributions, we will use the stronger versions of Symmetry and the Null-Player Axiom from Section 4. To gain extra intuition behind it, note that if $\alpha$-value still assigns zero to a null-player $i$, even if we remove player $j$ from the game (thus, $\varphi_{i}^{\alpha}\left(v_{-j}\right)=0$ ), then Balanced Contributions implies the Strong Null-Player Axiom: $\varphi_{j}^{\alpha}(v)-\varphi_{j}^{\alpha}\left(v_{-i}\right)=\varphi_{i}^{\alpha}(v)-$ $\varphi_{i}^{\alpha}\left(v_{-j}\right)=0-0$. On the other hand, the axiom of Balanced Contributions asks for the balance between the contributions of the two players contributions and ultimately implies Strong Symmetry.

Theorem 5 Shapley's marginality-based axiomatization (Efficiency, Symmetry, Additivity and $\alpha$-Null-Player Axiom) is equivalent to Myerson's axiomatization (Efficiency, $\alpha$-Balanced Contributions) if and only if $\alpha$ is interlace and expansion resistant.

Proof. Our proof is organized as follows: first we will argue that if $\alpha$ is interlace and expansion resistant then $\alpha$-value satisfies $\alpha$-Balanced Contributions. Then, we will prove that $\alpha$-Balanced Contributions implies the Strong Null-Player Axiom and Strong Symmetry. This result combined with Theorems 3 and 4 that link these axioms with properties of $\alpha$ will conclude the proof. Both parts of the proof will be based on the linear decomposition of the game $v$ to simple games $e^{(S, P)}$. If $\alpha$-Balanced Contributions is satisfied, then it must work for every simple game. On the other hand, if $\alpha$-Balanced Contributions works for every simple game then based on Additivity it must be satisfied also for every linear combination, thus every possible game $v$. Thus, $\alpha$-value satisfies $\alpha$-Balanced Contributions if and only if the following conditions are met:

(a) $\varphi_{i}\left(e^{(S, P)}\right)-0=\varphi_{j}\left(e^{(S, P)}\right)-0$ for $i, j \in S$; this condition comes directly from Symmetry;

(b) $\varphi_{i}\left(e^{(S, P)}\right)-\varphi_{i}\left(e^{\left(S, P_{-j}\right)} \cdot \alpha_{j}(S, P)\right)=\varphi_{j}\left(e^{(S, P)}\right)-0$ for $i \in S, j \notin S$;

(c) $\varphi_{i}\left(e^{(S, P)}\right)-\varphi_{i}\left(e^{\left(S, P_{-j}\right)} \cdot \alpha_{j}(S, P)\right)=\varphi_{j}\left(e^{(S, P)}\right)-\varphi_{j}\left(e^{(S, P-i)} \cdot \alpha_{i}(S, P)\right)$ for $i, j \notin S$.

The zeros in the equations come from the fact that game $e^{(S, P)}$ without player from $S$ is a zero game. A few times in our proofs we will use the following transformation:

$$
\varphi_{i}\left(e^{\left(S_{+j}, \tau_{j}^{S}(P)\right)} \cdot \alpha_{j}(S, P)\right)=\varphi_{j}\left(e^{\left(S_{+j}, \tau_{j}^{S}(P)\right)} \cdot \alpha_{j}(S, P)\right)=\varphi_{j}\left(e^{(S, P)}\right),
$$

for $i \in S, j \notin S$, where first transformation comes from Symmetry of players $i$ and $j$ (both players are in the only embedded coalition with the value), and second-from the $\alpha$-Null-Player Axiom.

First, assume $\alpha$ is interlace and expansion resistant (thus, $\alpha$-value satisfies Strong Symmetry and the Strong Null-Player Axiom). Consider condition (b). 
From the Strong Null-Player Axiom and Strong Symmetry $\varphi_{i}\left(e^{\left(S, P_{-j}\right)} \cdot \alpha_{j}(S, P)\right)=$ $\frac{|N|}{|S|} \varphi_{i}\left(e^{\left(S_{+j}, \tau_{j}^{S}(P)\right)} \cdot \alpha_{j}(S, P)\right)$ (this comes directly from formula $\left.(8)\right)$. From equation (10) this expression equals $\frac{|N|}{|S|} \varphi_{j}\left(e^{(S, P)}\right)$, thus condition (b) simplifies to $\varphi_{i}\left(e^{(S, P)}\right)=-\frac{|N|-|S|}{|S|} \varphi_{j}\left(e^{(S, P)}\right)$ which comes immediately from Strong Symmetry.

Now, let us focus on condition (c). Here, $\varphi_{i}\left(e^{(S, P)}\right)=\varphi_{j}\left(e^{(S, P)}\right)$ (from Strong Symmetry), thus condition simplifies to $\varphi_{i}\left(e^{(S, P-j)} \cdot \alpha_{j}(S, P)\right)=\varphi_{j}\left(e^{\left(S, P_{-i}\right)}\right.$. $\left.\alpha_{i}(S, P)\right)$. Again, we use the formula (8) to derive $\varphi_{i}\left(e^{\left(S, P_{-j}\right)} \cdot \alpha_{j}(S, P)\right)=$ $\frac{|N|}{|S|+1} \varphi_{i}\left(e^{\left(S_{+j}, \tau_{j}^{S}(P)\right)} \cdot \alpha_{j}(S, P)\right)=\frac{|N|}{|S|+1} \varphi_{i}\left(e^{\left(S_{+i j}, \tau_{i}^{S}\left(\tau_{j}^{S}(P)\right)\right)} \cdot \alpha_{j}(S, P) \cdot \alpha_{i}\left(S_{+j}, \tau_{j}^{S}(P)\right)\right)$ where the last transformation comes from the $\alpha$-Null-Player Axiom. From Symmetry and the interlace resistant property we can replace all $i$ with $j$ which is equal to the analogous transformation of the right-hand side of the equation.

Secondly, we will prove that Efficiency and $\alpha$-Balanced Contributions indeed imply Strong Symmetry and the Strong Null-Player Axiom. Let $\varrho(v)$ denote the number of players outside $S$ in the smallest coalition with a non-zero value, i.e., $\varrho(v)=\max _{(S, P): v(S, P) \neq 0} \sum_{T \in P, T \neq S}|T|$. If $v$ is a game of $N$ players, then $\sum_{T \in P, T \neq S}|T|=|N|-|S|$, but as we will consider games of various numbers of players, we define this number by the size of partition $P$. We will use the induction by $\varrho(v)$. If $\varrho(v)=0$ then $v=e^{(N,\{N, \emptyset\})}$ and both axioms are instantly satisfied. Now, assume that Strong Symmetry and the Strong Null-Player Axiom hold for games with $\varrho(v)<k$. We will prove that it holds also for games in which $\varrho(v)=k$. Let $(S, P)$ be an embedded coalition such that $\sum_{T \in P, T \neq S}|T|=k$. Then, Balanced Contributions implies $\varphi_{i}\left(e^{(S, P)}\right)-$ $\varphi_{i}\left(e^{(S, P-j)} \cdot \alpha_{j}(S, P)\right)=\varphi_{j}\left(e^{(S, P)}\right)$ for $i \in S, j \notin S$ (condition $\left.(\mathrm{b})\right)$. As $\varrho\left(e^{\left(S, P_{-j}\right)}\right.$. $\left.\alpha_{j}(S, P)\right)=k-1$, then from Strong Symmetry and the Strong Null-Player Axiom $\varphi_{i}\left(e^{\left(S, P_{-j}\right)}\right)=\frac{|N|}{|S|} \varphi_{i}\left(e^{\left(S_{+j}, \tau_{j}^{S}(P)\right)}\right)$ and using equation $(10)$ we have $\varphi_{i}\left(e^{(S, P)}\right)=$ $-\frac{|N|-|S|}{|S|} \varphi_{j}\left(e^{(S, P)}\right)$ which implies Strong Symmetry (payoff of player $j \notin S$ in game $e^{(S, P)}$ is equal for every $\left.j\right)$.

Now, we will prove that the Strong Null-Player Axiom is also satisfied. Assume that $i$ is a null-player in a strict sense. As argued in the proof of Theorem 4, game $v$ can be expressed as a linear combination of games of form $\tilde{v}^{(S, P)}=$ $e^{(S, P)}+\sum_{T \in P_{-S}} e^{\left(S_{-i}, \tau_{i}^{T}(P)\right)}$. It is enough to show that for every such game, $\varphi_{j}\left(\tilde{v}^{(S, P)}\right)=\varphi_{j}\left(\tilde{v}_{-i}^{(S, P)}\right)$ for every $j \in N$. If $j \in S$ this follows automatically from Balanced Contributions

$$
\varphi_{j}\left(\tilde{v}^{(S, P)}\right)-\varphi_{j}\left(\tilde{v}_{-i}^{(S, P)}\right)=\varphi_{i}\left(\tilde{v}^{(S, P)}\right)-\varphi_{i}\left(\tilde{v}_{-j}^{(S, P)}\right)=0-0 .
$$

And based on Strong Symmetry, the above is all we need. Specifically, for every $k \notin S$, it occurs that $\varphi_{k}\left(\tilde{v}^{(S, P)}\right)=\frac{|S|-1}{|N|-|S|} \varphi_{j}\left(\tilde{v}^{(S, P)}\right)$ and $\varphi_{k}\left(\tilde{v}_{-i}^{(S, P)}\right)=$ $\frac{|S|-1}{|N|-|S|} \varphi_{j}\left(\tilde{v}_{i}^{(S, P)}\right)$, thus $\varphi_{k}\left(\tilde{v}^{(S, P)}\right)-\varphi_{k}\left(\tilde{v}_{-i}^{(S, P)}\right)=0$. This concludes our proof. 


\section{THE ANALYSIS OF VARIOUS MARGINALITY DEFINITIONS}

In this section, we discuss the existing definitions of marginal contributions (i.e., $\alpha$ ) in the context of properties analyzed in Section 4. Before proceeding, let us recall that $P(i)$ denotes the coalition of player $i$ in a partition $P$.

We start with the externality-free marginality proposed by Pham Do and Norde [16]:

$$
\alpha_{i}^{\text {free }}(S, P)= \begin{cases}1 & \text { if } P(i)=\{i\} \\ 0 & \text { otherwise. }\end{cases}
$$

In other words, there exists only one transfer - that is forming a new coalitionwith non-zero weight. It is not difficult to notice that $\alpha^{\text {free }}$ is expansion resistant, because the weight of a transfer from $(S, P)$ does not depend on $S$. As far as the interlace resistance property is concerned, a product of weights will evaluate to 1 (the only non-zero value) if and only if the corresponding transfers always create a new coalition. Thus, the order of transfers does not have an impact on the value. This means that weights $\alpha^{\text {free }}$ are interlace resistant.

Skibski [18] used weights which are dual to the previous ones to derive McQuillin's value [11]:

$$
\alpha_{i}^{f u l l}(S, P)= \begin{cases}\frac{1}{\left|P_{-i}\right|-1} & \text { if } P(i) \neq\{i\} \text { or } P=\left\{N_{-i},\{i\}, \emptyset\right\} \\ 0 & \text { otherwise. }\end{cases}
$$

Here, the zero weight is associated with forming a new coalition (unless $i$ leaves the grand coalition and there are no coalitions to join) and transfers to all the other coalitions have equal probability. Analogously to the externality-free marginality, $\alpha^{\text {full }}$ is expansion resistant and also interlace resistant. To see this, let us consider a product of weights that corresponds to a given sequence of transfers. If any player forms a new coalition, the product evaluates to zero; if not, the size of the partition does not change and all weights equal $\frac{1}{\left|P_{-i}\right|-1}$. Clearly, in both cases the power of weights does not depend on the order of transfers.

Bolger's [2] definition of marginality is chronologically the first and perhaps the simplest:

$$
\alpha_{i}^{B}(S, P)=\frac{1}{\left|P_{-i}\right|} .
$$

It is based on an idea that for a given embedded coalition all transfers have the same weight. All weights are positive and do not depend on the size of $S$; thus, they satisfy the expansion resistance. Conversely, the interlace resistance is not satisfied, as creating a new coalition affects the weights of joining an existing coalition. For example, if we consider two transfers from coalition $\{1,2,3\}$ embedded in $(\{1,2,3\},\{4\}, \emptyset)$, one of 2 that forms a new coalition, and one of 3 that joins $\{4\}$, we have $\alpha_{3}^{B}(\{1,2\},\{\{1,2\},\{3,4\}, \emptyset\}) \cdot \alpha_{2}^{B}(\{1\},\{\{1\},\{2\},\{3,4\}, \emptyset\})=$ 
$\frac{1}{2} \frac{1}{2}$. But if the order were flipped we have $\alpha_{2}^{B}(\{1,3\},\{\{1,3\},\{2\},\{4\}, \emptyset\}) \cdot \alpha_{3}^{B}(\{1\},\{\{1\},\{2\},\{3,4\}, \emptyset\})=$ $\frac{1}{2} \frac{1}{3}$. Thus, $\operatorname{pr}_{4|| 2|| 3}^{\alpha^{B}}(\{1\},\{\{1\},\{2\},\{3,4\}, \emptyset\}) \neq \operatorname{pr}_{4|| 3|| 2}^{\alpha^{B}}(\{1\},\{\{1\},\{2\},\{3,4\}, \emptyset\})$.

Another concept, already mentioned in Section 3, was proposed by MachoStadler et al. [10]:

$$
\alpha_{i}^{M S}(S, P)=\frac{\left|P(i)_{-i}\right|}{\sum_{T \in P_{-S}}|T|}
$$

with the assumption that $|\emptyset|=1$. The authors assumed that weight of forming a new coalition is relatively small, but when such a coalition becomes bigger, the player is more likely to join it. Here, expansion resistance is satisfied, as $S$ is not counted in the denominator. Now, consider interlace resistance. We will prove that indeed $\alpha_{i}(S, P) \cdot \alpha_{j}\left(S_{+i}, \tau_{i}^{S}(P)\right)=\alpha_{j}(S, P) \cdot \alpha_{i}\left(S_{+j}, \tau_{j}^{S}(P)\right)$ for every embedded coalition $(S, P)$ such that $i, j \notin S$ (based on Lemma 4 this condition is sufficient). The product of the denominators (which simply increases by one after every transfer) appears in the formula on both sides. On the other hand, the numerator equals $(|P(i)|-1)(|P(j)|-1)$ (and $(|P(i)|-1)(|P(i)|-2)$ if both players are in the same coalition) regardless of the order of players.

The last marginality was proposed by $\mathrm{Hu}$ and Yang [9] and has been already mentioned in Section 4.1. It is defined as follows: ${ }^{13}$

$$
\alpha_{i}^{H Y}(S, P)=\frac{\left|\left\{R \in \mathcal{P}(N): R_{[S]}=P_{[S]}\right\}\right|}{\mid\left\{R \in \mathcal{P}(N): R_{[S \cup\{i\}]}=P_{[S \cup\{i\}]} \mid\right.}
$$

Intuitively, the numerator equals the number of partitions that contain the same partition of players $N \backslash S$. In the denominator, we assume that $i$ has not left $S$ yet-we count partitions that contain the same partition of players $N \backslash(S \cup\{i\})$. This marginality is not resistant to expansion, as the size of the $S$ affects the proportion between the numerator and the denominator. For example, $\alpha_{3}^{H Y}(\{1\},\{\{1\},\{2\},\{3\}, \emptyset\})=\frac{3}{5}$, and $\alpha_{3}^{H Y}(\{1,4\},\{\{1,4\},\{2\},\{3\}, \emptyset\})=$ $\frac{10}{15}$. On the other hand, $\mathrm{Hu}$ and Yang's marginality satisfies interlace resistance: product of two consecutive weights $\alpha_{i}(S, P) \cdot \alpha_{j}\left(S_{+i}, \tau_{i}^{S}(P)\right)$ simplifies to $\frac{\left|\left\{R \in \mathcal{P}(N): R_{-S}=P_{-S}\right\}\right|}{\left\{\left\{R \in \mathcal{P}(N): R_{-(S \cup\{i, j\})}=P_{-(S \cup\{i, j\})}\right\} \mid\right.}$ regardless of order of $i$ and $j$ (again, we used Lemma 4).

All the above observations are summarized in Table I. As we can see, most of the definitions of marginality satisfy our properties of the interlace and expansion resistance. The only value that meets all four properties - that is Weak and Strong Monotonicity, Strong Symmetry and the Strong Null-Player Axiom-is the value proposed by Macho-Stadler et al.

Finally, we address the value proposed by Myerson [12]. It also satisfies Shapley's axioms and can be derived using the marginality approach. However, Myerson's axiomatization based on the concept of carrier is far from the marginality analysis and results in a complex weights that do not meet any of the

\footnotetext{
${ }^{13}$ Note that here $N$ is the set of all players in $P$. As we consider $\alpha$ for $\left(S_{-j}, P_{-j}\right)$ set $N$ changes.
} 


\begin{tabular}{l|c|c|c|c} 
& non-negative & positive & interlace resistant & expansion resistant \\
\hline Bolger & $\checkmark$ & $\checkmark$ & - & $\checkmark$ \\
Pham Do \& Norde & $\checkmark$ & - & $\checkmark$ & $\checkmark$ \\
Skibski & $\checkmark$ & - & $\checkmark$ & $\checkmark$ \\
Hu \& Yang & $\checkmark$ & $\checkmark$ & $\checkmark$ & $\checkmark$ \\
Macho-Stadler et al. & $\checkmark$ & $\checkmark$ & $\checkmark$ &
\end{tabular}

THE PROPERTIES OF EXISTING WEIGHTS IN THE MARGINALITY APPROACH.

four properties. For instance, for $N=\{1,2,3\}$ and an embedded coalition $(\{1,2\},\{\{1,2\},\{3\}, \emptyset\})$ the weight of transfer of 1 to a new coalitions equals $\alpha_{1}(\{2\},\{\{1\},\{2\},\{3\}, \emptyset\})=2$, while joining player 3: $\alpha_{1}(\{2\},\{\{1,3\},\{2\}, \emptyset\})=$ -1 .

\section{RELATED WORK}

We divide the works on extending the concept of the Shapley value to games with externalities into three bodies of literature. First, we discuss the marginalitybased axiomatizations of values that satisfy Shapley's axioms. Next, we present other axiomatizations that yield values satisfying Shapley's axioms. Finally, we briefly address all the values that violate Shapley's axioms.

First, three works proposed new definitions of marginality and proved uniqueness based on Shapley's standard axiomatization [16, 9, 18]. Pham Do and Norde, and $\mathrm{Hu}$ and Yang proposed new values, while Skibski provided a marginal axiomatization for McQuillin's value (discussed below). These uniqueness results are the special cases of our Theorem 1. Some other authors used Young's axiomatization-Bolger modified it by adding an additional Null-Player Axiom to derive his value [2]; and De Clippel and Serrano in their analysis of externalityfree value [3]. These results for Young's axiomatization were generalized by Fujinaka [6]. He was the first to propose a general formula for marginal contribution as the affine combination of elementary marginal contributions. Fujinaka proved that Young's axiomatization parametrized by any weights $\alpha$ implies a unique value. Our Theorem 1 is the equivalent of Fujinaka's result but for Shapley's axiomatization.

Macho-Stadler, Perez-Castrillo and Wettstein [10] proposed the average approach that was discussed in detail in Section 4.3, where we showed that it is equivalent to the marginality approach with interlace resistant weights (see Corollary 1$)^{14}$. Using the average approach, the authors provided a value using Shapley's axioms together with Strong Symmetry (see Section 4.3) and Similar Influence. This latter axiom says that, if we exchange the values of two embedded coalitions in which players $i$ and $j$ appear in the first one together and, in

\footnotetext{
${ }^{14}$ We note that a one-way proof for positive weights was already presented by $\mathrm{Hu}$ and Yang [9]. More precisely, they proved that every value obtained using average approach with positive weights can be derived using marginality approach.
} 
the second one, as singletons, then their payoffs should not change. Although axiomatization departed from marginality, the authors introduce a definition of marginal contribution and note that value can be transformed as the weighted average of player's marginal contributions.

McQuillin [11] analyzed extending the Shapley value to games with externalities combined with Owen generalization [14]. If we specify how the payoffs of players should be generalized for payoffs of coalition (to this end, McQuillin provided a Rule of Generalization), then we can treat the payoffs as a game (as all coalitions have assigned payoffs). McQuillin argued that stability is be reached when a given payoff is a fixed-point of this process (i.e. if we consider a value to be a game by itself, then the value computed for such a game should be the same). McQuillin called this requirement Recursion and proved that combined with Rules of Generalization, Weak Monotonicity and Shapley's axioms implies a unique value.

Myerson was the first to propose a new extension of the Shapley value to games in a partition-function form. He based his value on the concept of Carrier. We say that a set $C$ is a carrier if the value of any embedded coalition is determined by a partition of players from $C$. Now, Carrier implies that, if $C$ is a carrier, then the payoff of the grand coalition is divided between players from $C$. Against this, Myerson showed that there exists a unique value that satisfies Symmetry, Additivity and Carrier. As the set of all players, $N$, is clearly a carrier and, if $i$ is a null-player, then $N \backslash\{i\}$ is also a carrier, we have that Carrier implies both Efficiency and the Null-Player Axiom. This means that Myerson's value satisfies all four of Shapley's axioms.

Other authors proposed values that are rather far from Shapley's understanding of fairness.

Albizuri et al. [1] argued that, in a game with externalities, a coalition should be evaluated by the set of values it has, regardless of which partitions these values correspond to. The authors combined this principle, called Embedded Coalition Anonymity, with The Oligarchy Axiom (which can be understood as the weakened Myerson axiom) and three of Shapley's original axioms: Efficiency, Additivity and Symmetry. The resulting value can be derived as the Shapley value for a game without externalities calculated by assigning to every coalition an arithmetic average of all its values in games with externalities. Although, at first, it seems like a special case of the average approach, proposed weights violates the condition necessary to satisfy the Null-Player Axiom (see Theorem 1 in $[10])$.

In a stochastic process described by us in Section 3, players leave the grand coalition one by one. Grabisch and Funaki [7] formulate a different process. They take as a starting point the partition containing singletons of all players and consider all possible sequences of mergers which result in the grand coalition. That said, the contribution of a player is evaluated as the effect that the player merging with other coalitions makes on their values. If a player enters some coalition alone, he is rewarded with the whole change of its value, i.e., with 
the marginal contribution; but if he is already a part of a coalition that merges with another one, Grabisch and Funaki argue that the change of the value of the coalition they merge with should be divided equally between him and other members of the coalition. This contradicts the Null-Player Axiom, as a nullplayer is rewarded with a payoff even though the coalition without him would cause the same impact on the merged coalition.

Finally, let us address the concepts proposed by Maskin [4] and Hafalir [8]. Here, the authors discarded the assumption that the grand coalition will form and proposed to divide the payoff of optimal coalition structure. Maskin studied the coalition formation process and proposed an axiomatic characterization of a value expected in this process. Hafalir proposed a mechanism that implements a unique payoff division and provided axiomatization based on the idea of efficientcover. These ideas, although interesting, result in an axiomatization significantly different to Shapley's.

\section{CONCLUSIONS}

We were concerned in this paper with the issue of deriving the value for games with externalities. We studied the marginality approach to this problem which is based on Shapley's axiomatization, where the Null-Player Axiom is strengthened by assuming that marginal contribution is the affine combination of elementary marginal contributions, denoted $\alpha$.

We proved that the marginality approach yields a unique value for every $\alpha$. Furthermore, we showed that all values that satisfy the direct translation of Shapley's axioms can be obtained using the marginality approach. We then studied various properties of a value and how they translate to the requirements of the definition of the marginal contribution. In particular, we first focused on Weak and Strong Monotonicity and showed that an $\alpha$-value satisfies the first (second) axioms if and only if significant weights $\alpha$ are non-negative (positive). Then we analyzed Strong Symmetry and the Strong Null-Player Axiom and proved that $\alpha$-value satisfies Strong Symmetry if and only if $\alpha$-marginality is interlace resistant; and, if $\alpha$ is interlace resistant, it satisfies the Strong Null-Player Axiom if and only if $\alpha$-marginality is expansion resistant. Building upon this analysis we demonstrated that Myerson's axiomatization for games with externalities is equivalent to Shapley's axiomatization if and only if $\alpha$ is interlace and expansion resistant.

Our work can be extended in various directions. We concentrated on the properties of Strong Symmetry and the Strong Null-Player Axiom as crucial for Myerson's axiomatization, but other links between the axioms met by values and weights property can be sought. Another interesting question is how big the set of Strong Monotonic values is that satisfies both Strong Symmetry and the Strong Null-Player Axiom. To date, there has been only one such solution found. Furthermore, with our intuition based on the stochastic processes, we hint at some relations between the extension of the Shapley value and the probability theory. 
Can the choice of the suitable value for a given application be driven by the specific probabilities of merging groups in a given environment? This question is left for our future work.

\section{ACKNOWLEDGEMENTS}

Tomasz Michalak and Michael Wooldridge were supported by the European Research Council under Advanced Grant 291528 ("RACE"). We would like to thank Andrzej Szalas for various comments and suggestions.

\section{REFERENCES}

[1] M. Albizuri, J. Arin, and J. Rubio. An axiom system for a value for games in partition function form. Int. Game Theory Review, 7(01):63-72, 2005.

[2] E. Bolger. A set of axioms for a value for partition function games. International Journal of Game Theory, 18(1):37-44, 1989.

[3] G. de Clippel and R. Serrano. Marginal contributions and externalities in the value. Econometrica, 76(6):1413-1436, 2008.

[4] E. E. Maskin. Bargaining, coalitions, and externalities. presidential address of the econometric society, 2003.

[5] U. Faigle and M. Grabisch. A note on values for markovian coalition processes. Economic Theory Bulletin, pages 1-12, 2013.

[6] Y. Fujinaka. On the marginality principle in partition function form games. Unpublished manuscript, 2004.

[7] M. Grabisch and Y. Funaki. A coalition formation value for games with externalities. Technical Report b08076, Universit Panthon-Sorbonne (Paris 1), Centre d'Economie de la Sorbonne, 2008.

[8] I. Hafalir. Efficiency in coalition games with externalities. Games and Economic Behavior, 61(2):242-258, 2007.

[9] C.-C. Hu and Y.-Y. Yang. An axiomatic characterization of a value for games in partition function form. SERIEs, 1(4):475-487, 2010.

[10] I. Macho-Stadler, D. Perez-Castrillo, and D. Wettstein. Sharing the surplus: An extension of the shapley value for environments with externalities. Journal of Economic Theory, 135(1):339-356, 2007.

[11] B. McQuillin. The extended and generalized shapley value: Simultaneous consideration of coalitional externalities and coalitional structure. Journal of Economic Theory, 144(2):696-721, 2009.

[12] R. Myerson. Values of games in partition function form. International Journal of Game Theory, 6:23-31, 1977.

[13] R. Myerson. Conference structures and fair allocation rules. International Journal of Game Theory, 9:169-82, 1980.

[14] G. Owen. Values of games with a priori unions. In Mathematical economics and game theory, pages 76-88. Lecture Notes in Econ. and Math. Systems, Vol. 141. Springer, Berlin, 1977. 
[15] D. Pérez-Castrillo and D. Wettstein. Bidding for the surplus: A noncooperative approach to the shapley value. Journal of Economic Theory, $100(2): 274-294,2001$.

[16] K. PhamDo and H. Norde. The shapley value for partition function form games. International Game Theory Review, 9(02):353-360, 2007.

[17] L. Shapley. A value for n-person games. In H. Kuhn and A. Tucker, editors, Contributions to the Theory of Games, volume II, pages 307-317. Princeton University Press, 1953.

[18] O. Skibski. Steady marginality: A uniform approach to shapley value for games with externalities. In Symposium on Algorithmic Game Theory, volume 6982 of $L N C S$, pages 130-142. Springer, 2011.

[19] P. Young. Monotonic solutions of cooperative games. International Journal of Game Theory, 14(2):65-72, 1985. 